\title{
INDEX OF New Names OF SynTAXA Published IN 1994
}

\author{
Jean-Paul Theurillat ${ }^{1)}$ \& Jaroslav Moravec ${ }^{2)}$ \\ 1) Centre alpien de Phytogéographie, Fondation J.-M. Aubert, CH-1938 Champex, and Conservatoire et \\ Jardin botaniques, Case postale 60, CH-1292 Chambésy, Switzerland; fax +4122418 5101, \\ E-mail theurillat@cjb.unige.ch \\ 2) Institute of Botany, Academy of Sciences of the Czech Republic, CZ-252 43 Prühonice, Czech Republic; \\ fax +420267750031
}

Keywords: Alliances, Associations, Classes, Code of Phytosociological Nomenclature, Nomina corrigenda, Nomina invalida, Nomina inversa, Nomina mutata, Nomina nova, Orders, Phytosociology

\begin{abstract}
The present work collects the new names of syntaxa (in the sense of the Code of phytosociological nomenclature, BARKMAN et al. 1986) above subassociation rank found in the literature received by the Library of the Conservatoire Botanique in Geneva. For the year 1994851 names were listed corresponding to 34 classes, 55 orders, 2 suborders, 128 alliances, 29 suballiances, and 603 associations. For each of them, an assessment of its validity is given relating to the Code of Phytosociological Nomenclature. 72 names are given to add to the Indices 1991, 1992, 1993 (ThEuRILLAT \& MORAVEC 1994, 1995, 1996).
\end{abstract}

Pursuing our work started in 1987 (THEURILLAT \& MORAVEC 1990) on indexing new names of syntaxa published yearly, we present below the "Index 1994", containing 851 names of syntaxa higher than the subassociation rank (sensu BARKMAN et al. 1986). Furthermore, one will find in the addendum 33 names to the Index 1991 (THEURILLAT \& MORAVEC 1994), 18 to the "Index 1992" (THEURILlAT \& MORAVEC 1995) and 21 names to the "Index 1993" (THEURILLAT \& MORAVEC 1996). These names were compiled mainly following consultation of the literature at the library of the Conservatoire et Jardin botaniques of the city of Geneva.

The "Index" follows the same principles as the "Index 1987", "Index 1989" and "Index 1992" (THEURILLAT \& MORAVEC 1990, 1992, 1995) to which reference will be made. As previously, the classes are listed in alphabetical order and, in each class, syntaxa (order, alliance, association) are listed in the same way, the communities of algae, lichens and bryophytes being separated from the communities of vascular plants. The symbols $(0,0, *$, $\Delta$ ) preceding the names indicate the nomenclatural validity. Validly published names are preceded by - and invalidly published or illegitimate ones by $O$. The asterisk (*) indicates superfluous names and doubtful cases requiring further bibliographical or nomenclatural investigations. For the latter, the main reasons are given. The category of "unchecked names" $(\Delta)$, introduced in the "Index 1993", includes for verification those names whose validity could not be readily verified because the required bibliographical references were not available to the authors. In this way they are removed from the category of doubtful names in which they were previously included.

The geographical location of associations (and in some cases of syntaxa of higher ranks) whenever this information was directly and clearly available, is placed at the end of the entry. Location is given according to the principles of the system of the "World geographical scheme for recording plant distributions" (HOLLIS \& BRUMMITT 1992, see THEURILLAT \& MORAVEC 
1995). As a matter of simplification and clarity, the only abbreviations used are the two-letter International Standards Organisation codes (ISO Standard 3166), which often differ from postal codes or number plates. The locations which do not follow the "Scheme" are cited between quotation marks (e.g. "Alps"). As a rule, nomenclature of locations follows "The Times Atlas".

The classes are retained independently of their nomenclatural validity. Quotation marks indicate that the name of the class used is, very likely, invalidly published (e.g. "Xanthostemo-Podocarpetea novae-cadeloniae", "Abrotanello-Azorelletea selaginis", "Acacio-Styphelietea cymbulae", "Aglaio-Cupaniopsietea glomeriflorae", "Avicennio-Salicornietea australis", etc.). In cases where syntaxonomic knowledge does not allow attachment of a name to a class, the highest syntaxonomical rank known is retained, i.e. for the communities of vascular plants Diheteropogono amplectentis-Schizachyrion sanguinei, Loudetio simplicis-Schizachyrietalia sanguinei, Lupino-Calamagrostion tolucensis, Protosparago africani-Acacion karroo, Rhoo leptodictyo-Acacion caffrae, Trachypogono spicati-Diheteropogonion amplectentis.

The 851 names published in 1994 are divided into 127 classes and 8 other ranks, and include the two groups Incertae sedis. These 851 new names correspond to 34 classes, 55 orders, 2 suborders, 128 alliances, 29 suballiances, and 603 associations. There are 23 classes containing 10 or more names, including altogether 360 names (42\%), among them Asteretea tripolii with 32 names, Festuco-Brometea with 29, Potametea with 33, Querco-Fagetea with 21. Under the headings Incertae sedis 92 names (11\%) are listed.

Regarding previous "Indices", we would like to point out to the authors the high percentage of invalid or illegitimate names, i.e. 440 (48\%) for the "Index 1994" with the additions. Although provisional names (art. 3b), the application of which is usual in cases where the syntaxon in question is not sufficiently known, correspond to more than the half of the invalid and illegitimate names, we would again ask all authors to conform more closely to the Code of phytosociological nomenclature (BARKMAN et al. 1986). This situation is also of concern because of the very high percentage of invalid names of higher ranks: 31 classes out of 34 , 48 orders out of 55, and 92 alliances out of 128 were invalidly published in 1994. Invalid and illegitimate names are counter-productive in phytosociological literature. Therefore, we would also ask the editors of phytosociological publications to pay more attention to the nomenclatural aspects of the articles they publish. In our opinion, conformity with the Code is as important as the quality of the data or of their analysis.

Names corrected according to art. 42 (nomina inversa) or art. 45 (nomina mutata), when published by individual authors, are considered illegitimate (i.e. not published in accordance with the Code). In such cases we would like to invite the authors to propose the correction of the names, giving reasons, to the Nomenclatural Commission (addressed to the Secretary). For the acceptance of a nomen inversum the dominance of the name-giving species in the original diagnosis is decisive. For the acceptance of a nomen mutatum it must be proved that the name of the name-giving species has not been used in the taxonomic literature for at least 20 years as the correct name. The same is true for proposals of nomina ambigua according to art. 36. In the meantime, proposals of inversion or mutation can be used only when they are indicated specifically as such (e.g. nom. invers. propos., nom. mut. propos.; see THEURILLAT 1997).

For the 440 invalid or illegitimate names of the "Index 1994" (additions included), the causes of invalidity are (1) provisional names (art. 3b) (256 names i.e. 58\% of all the cases), 
(2) no type given to the name (art. 5) (85 names i.e. 19\%), (3) inadequacy in the original diagnosis or a lack of bibliographical reference to a sufficient diagnosis (art. 2b, 8) (57 names i.e. $13 \%$ ), (4) the taxon's name forming the syntaxon's names is unclear (art. $3 \mathrm{~g}$ ) or the name-giving taxon is absent from the diagnosis (art. 3f) (17 names, i.e. 4\%), (5) the indicated rank does not correspond to ranks of the Code (art. $3 \mathrm{~d}$ ), (13 names, i.e. 3\%), (6) non conformity to the form of the name (art. $3 \mathrm{~h}, 14)$ ( 3 names, i.e. $1 \%)$. The causes of illegitimacy ( 9 names i.e. $2 \%$ of all cases) relate to corrections of the names (art. 30) (1 name), to homonymy (art. 31) (2 names), to nomina inversa (art. 42) (2 names), and to nomina mutata (art. 45) (4 names).

In addition to the 440 invalid or illegitimate names, there are 10 doubtful names (*), of which 7 are superfluous names resulting from unnecessary corrections of names (art. 29), and 21 unchecked names $(\Delta)$.

In order to draw up as complete a list as possible in the future, the authors welcome receipt of missing elements in the form of offprints. Any comments regarding nomenclature would also be appreciated.

\section{INDEX 1994}

\section{Communities of cryptogams}

Barbuletea unguiculatae

$\Delta$ Gymnostomo viriduli-Southbyetum nigrellae GuerRA et Gll in Ríos et GuerRA 1987 corr. MoYA, Ríos et GUERRA $1994(: 47)$

- Tortuletum parnassicae FREY et KÜRSCHNER 1994 ass. nov. (: 369) [JO]

Cystoseiretea

$\Delta$ Cladophoro-Rhytiphloetum tinctoriae GIACCONE in GIACCONE, Alongl, PIzzuTo et Cossu 1994 [1995?] ass. nov. (: 137) [IT]

$\Delta$ Cystoseiretum sauvageauanae GiaCCONE in GiacCone, Alongi, PizzUTo et Cossu 1994 [1995?] ass. nov. (: 124) [IT]

Grimmietea anodontis

- Grimmietum teretinervis VADAM 1994 ass. nov. (: 157) [FR]

- Tortuletum caninervis FrEY et KÜRSCHNER 1994 ass. nov. (: 375) [JO]

Rhizocarpetea geographici

- Lecanoro sulphurellae-Ramalinetum clementeanae EGEA et LLIMONA 1994 ass. nov. (: 275) [ES]

Tortulo-Homalothecietea sericei

O Homalothecio sericei-Leptodontetum smithii MOYA, Ríos et GUERRA 1994 ass. nov., nom. inval. (: 49) [art. 5] [ES]

"Xanthostemo-Podocarpetea novae-caledoniae"

O Xanthemostemo-Podocarpetea novae-caledoniae Hoff $1994 \mathrm{cl}$. nov., nom. prov. (: 682) [art. 3b] [NC]

O Xanthemostemo-Podocarpetalia novae-caledoniae HoFf 1994 ord. nov., nom. prov. (: 682) [art. 3b] [NC]

O Xanthemostemo-Podocarpion novae-caledoniae HofF 1994 all. nov., nom. prov. (: 682) [art. 3b] [NC]

O Caryophyllo-Syzygietum ngoyensis HoFf 1994 ass. nov., nom. prov. (: 682) [art. 3b] [NC]

Incertae sedis

- Ramonio subsphaeroidis-Striguletum mediterraneae BRICAUD et RovX 1994 ass. nov. (: 118, 126) [FR]

- Striguletum affinis BRICAUD et RoUX 1994 ass. nov. (: 129, 130) [FR] 


\section{Communities of vascular plants}

"Abrotanello-Azorelletea selaginis"

O Abrotanello-Azorelletea selaginis PISANO $1994 \mathrm{cl}$ nov., nom. prov. (: 12, 18) [art. 3b] [CL, Chile South]

O Abrotanello-Bolacetalia gummiferae PISANO 1994 ord. nov., nom. prov. (: 12, 18) [art. 3b] [CL. Chile South]

O Caltho-Abrotanelletalia PISANO 1994 ord. nov., nom. prov. $(: 12,19)$ [art. 3b] [CL, Chile South]

O Abrotanello-Bolacion gummiferae PISANO 1994 all. nov., nom. prov. (: 12) [art. 3b] [CL, Chile South]

O Caltho-Abrotanellion PISANo 1994 all. nov., nom. prov. (: 12) [art. 3b] [CL, Chile South]

O Empetro-Pernettyion PISANO 1994 all. nov., nom. prov. (: 12) [art. 3b] [CL, Chile South]

O Caltho-Abrotanelletum PISANo 1994 ass. nov., nom. prov. (: 12) [art. 3b] [CL, Chile South]

O Empetro-Azorelletum lycopodioidis PISANO 1994 ass. nov., nom. prov. (: 12, 18) [art. 3b] [CL, Chile South]

O Festuco-Bolacetum gummiferae PISANO 1994 ass. nov., nom. prov. $(: 12,18)$ [art. 3b] [CL, Chile South]

O Usneo-Polystichetum andinae PISANo 1994 ass. nov., nom. prov. (: 12, 19) [art. 3b] [CL, Chile South]

"Acacio-Styphelietea cymbulae"

O Acacio-Styphelietea cymbulae HoFf $1994 \mathrm{cl}$. nov., nom. prov. (: 681) [art. 3b] [NC]

O Acacio-Styphelietalia cymbulae HoFf 1994 ord. nov., nom. prov. (: 681) [art. 3b] [NC]

O Acacio-Grevillion meisneri HoFf 1994 all. nov., nom. prov. (: 681) [art. 3b] [NC]

O Baumeo-Maxwellion lepidotae HoFf 1994 all. nov., nom. prov. (: 681) [art. 3b] [NC]

O Solano-Bocquillonion spinuligerae HoFf 1994 all. nov., nom. prov. (: 681) [art. 3b] [NC]

O Acacio-Solanetum styraciflori HoFf 1994 ass. nov., nom. prov. (: 681) [art. 3b] [NC]

O Cloezio-Maxwellietum angustifoliae HoFf 1994 ass. nov., nom. prov. (: 681) [art. 3b] [NC]

O Cloezio-Serianthetum germainii HofF 1994 ass. nov., nom. prov. (: 681) [art. 3b] [NC]

O Euroschino-Syzygietum austrocaledonicae Hoff 1994 ass. nov., nom. prov. (: 681) [art. 3b] [NC]

O Gahnio-Jasminetum artensi HoFf 1994 ass. nov., nom. prov. (: 681) [art. 3b] [NC]

O Garcinio-Atractocarpetum rotundifolii HoFF 1994 ass. nov., nom. prov. (: 681) [art. 3b] [NC]

O Wikstroemio-Lepidospermetum perteretis HOFF 1994 ass. nov., nom. prov. (: 681) [art. 3b] [NC]

O Xanthostemo-Ixoretum collinae HoF 1994 ass. nov., nom. prov. (: 681) [art. 3b] [NC]

Adiantetea

- Homalio lusitanicae-Adiantetum PUGLisi 1994 [1995?] ass. nov. (: 95) [IT, Sicilia]

"Aglaio-Cupaniopsietea glomeriflorae"

O Aglaio-Cupaniopsietea glomeriflorae HoF $1994 \mathrm{cl}$ nov., nom. prov. (: 680) [art. 3b] [NC]

O Aglaio-Cupaniopsietalia glomeriflorae Hoff 1994 ord. nov., nom. prov. (: 680) [art. 3b] [NC]

O Arythero-Alyxietalia celastrinae HoFF 1994 ord. nov., nom. prov. (: 680) [art. 3b] [NC]

O Arythero-Gardenion urvillei HoFF 1994 all. nov., nom. prov. (: 680) [art. 3b] [NC]

O Elaeodendro-Aglaion eleagnoidis HoFF 1994 all, nov., nom. prov. (: 680) [art. 3b] [NC]

O Jasmino-Mabion buxifoliae HOFF 1994 all. nov., nom. prov. (: 680) [art. 3b] [NC]

O Microsorio-Schefflerion golip HOFF 1994 all. nov., nom. prov. (: 680) [art. 3b] [NC]

O Aglaio-Cupaniopsietum glomeriflorae HoFf 1994 ass. nov., nom. prov. (: 680) [art. 3b] [NC]

O Cryptocaryo-Scheffleretum golip HoF 1994 ass. nov., nom. prov. (: 680) [art. 3b] [NC]

O Delarbreo-Microsorietum punctati HoFf 1994 ass. nov., nom. prov. (: 680) [art. 3b] [NC]

O Diospyro-Jasminetum leratii HoFF 1994 ass. nov., nom. prov. (: 680) [art. 3b] [NC]

O Dysoxylo-Oleetum paniculatae HoFf 1994 ass. nov., nom. prov. (: 680) [art. 3b] [NC]

O Jasmino-Mabetum buxifoliae HoFF 1994 ass. nov., nom. prov. (: 680) [art. 3b] [NC]

O Mabo-Podonephelietum homei HoFf 1994 ass. nov., nom. prov. (: 680) [art. 3b] [NC]

Agropyretea intermidii-repentis [see Artemisietea]

Agrostietea stoloniferae

- Echinodoro-Paspaletum distichi GÉHU, KAABECHE et GHARZOULI 1994c ass. nov. (: 303) [DZ]

- Eryngio barrelieri-Caricetum divisae GÉHU, KAABECHE et GHARZOUL1 1994c ass. nov. (: 301) [DZ] 
- Helosciadio crassipedis.Paspaletum distichi GÉHU, KAABECHE el GHARZOULI 1994c ass, nov. (: 301) [DZ]

Alnetea glutinosae

- Carici lusitanicae-Alnetum glutinosae DÍAZ GONZÁleZ et FERNÁNDEZ PRIETo 1994 ass. nov. (: 313) [ES]

- Rusco hypophylli-Alnetum glutinosae GÉHU, KAABECHE et GHARZOULI 1994b ass. nov. (: 69) [DZ]

\section{Artemisietea}

- Anthemido-Centaureetum rhenanae DENGLER 1994 ass. nov. (: 291) [DE]

- Carduo cephalanthi-Dracunculetum muscivori GAMISANS et PARADIS ex GÉHU et BIONDI 1994 ass. nov. (: 126) [FR, Corse]

- Convolvulo arvensis-Caricetum hirtae JEHLík 1994 ass. nov. (: 261)

- Urtico-Lamietum orvalae ČARN 1994 ass. nov. (: 490) [SI]

Artemisietea lerchianae

- Artemisietea lerchianae Golub 1994b cl. nov. (: S00) [RU, Russia South]

- Artemisietalia lerchianae Golub 1994b ord. nov. (: 500) [RU, Russia South]

- Artemision lerchianae Golub 1994b all. nov. (: 500) [RU, Russia South]

- Anabasietum aphyllae Golub $1994 b$ ass. nov. (: 504) [RU, Russia South]

- Kochietum prostratae GOLUB 1994b ass. nov. (: 500) [RU, Russia South]

- Salsoletum dendroidis Golub 1994b ass. nov. (: 503, 505) [RU, Russia South]

Artemisietea tschernievianae

O Artemisietea tschernievianae GOLUB 1994b cl. nov., nom. inval. (: 505) [art. 8] [RU, Russia South]

O Antemisietalia tschernievianae GOLUB 1994b ord. nov., nom. inval. (: 505) [art. 5] [RU, Russia South]

- Euphorbion seguierianae Golub 1994b all. nov. (: 509) [RU, Russia South]

- Tamarici-Salsolion australis GOLUB 1994b all. nov. (: 509, 510) [RU, Russia South]

- Artemisietum tschernievianae GoluB 1994b ass. nov. (: 509) [RU, Russia South]

- Koelerietum sabuletori Golub 1994 b ass. nov. (: 509) [RU, Russia South]

- Salsolo australis-Atriplicetum tataricae GoLUB 1994b ass. nov. (: 511, 512) [RU, Russia South]

- Salsolo australis-Cannabetum ruderalis GoluB 1994b ass. nov. (: 512, 513) [RU, Russia South]

- Tamarici-Salsoletum australis GoluB 1994b ass. nov. (: 513) [RU, Russia South]

- Zygophyllo fontanesii-Suaedetum verae BIONDI, AllEgreZZA, TAFFETANl et WiLdPRET DE LA TORRE 1994 ass. nov. (: 111$)[E S]$

Asplenietea trichomanis

- Artemisio nitidae-Silenetum lanuginosae TOMASELLI 1994 ass. nov. (: 40, 46) [IT]

- Asplenio rutae-murariae-Gymnocarpietum robertiani KOLBEK et SÁDLO 1994 ass. nov. (: 126, 128) [CZ]

- Dauco hispanici-Asplenietum marini Gamisans et PARAdIS ex GEHU et BIONDI 1994 ass. nov. (: 90) [FR, Corse]

- Diantho sylvestris-Seselietum praecocis LAMBinon et PIRONET ex GÉHU et BIONDi 1994 ass. nov. (: 91) [FR, Corse]

- Drabo aizoidis-Primuletum apenninae TOMASELll 1994 ass. nov. $(: 41,47)$ [IT]

- Dryopterido aemulae-Hymenophylletum tunbrigensis DÍAZ GONZÁLEZ et FERNÁNDEZ PRIETO 1994 ass. nov. (: 439$)$ [ES]

- Phagnalo sordidi-Jasionetum mansanetianae Roselló GIMENo 1994 ass, nov. (: 389) [ES]

- Silenetum saxifragae TOMASELLI 1994 ass. nov. (: 40, 46) [IT]

- Umbilico rupestris-Asplenietum obovati BIONDI, BOCCHIERI, BRUGIAPAGLIA et Mulas ex GÉHU et BIONDI 1994 ass. nov. (: 91) [FR, Corse]

Asteretea tripolii

O Cirsietalia esculenti MIRKIN et Golub in Golub 1994a ord. nov. (: 25) [art. 5, 17] [UA. RU, Russia Central, Russia East, Western Siberia]

- Halerpestetalia MiRKIN, NAUMOVA et SOlOMESTCH ex Golvb 1994a ord. nov. (: 42) [MN, RU, Yakutiya]

- Suaedetalia corniculatae GolvB 1994a ord. nov. (: 46) [MN, RU, Yakutiya] 
Artemisienalia santonicae GoluB 1994a subord. nov, (: 36) [UA, RU]

O Elytrigienalia repentis GoluB 1994a subord. nov., nom. inval. (: 33) [art. 5, 17] [RU]

- Alhagion Golub et TCHORBADzE in Golub 1994a all. nov. (: 37) [RU, Russia South]

- Cirsio-Hordeion MiRKiN ex GoluB 1994a all. nov. (: 32) [RU, Russia East]

- Cirsion esculenti Golub 1994a all. nov., nom. inval. (: 32) [art. 3g] [RU, Russia East, Western Siberia]

- Geranion collini Golub et SAVELJEva in Golub 1994a all. nov. (: 25) [RU, Russia Central, Russia East, Russia South, Western Siberia]

- Halerpestion salsuginosae MiRkin, Naumova et Solomestch ex Golub 1994a all. nov. (: 46) [MN]

- Limonion gmelinii Golub 1994a all. nov. (: 33) [RU, Russia Central, Russia East, Russia South, Western Siberia]

O Limonion sareptani Golub 1994a all. nov., nom. inval. (: 33) [art. 3f] [RU, Russia South]

- Limonion tomentelli AGAFONOV et Golub in Golub 1994a all, nov. (: 36) [RU, Russia Central]

- Puccinellion fominii SHelyaG-SosonKo et SolomaKHA ex Golub 1994a all. nov. (: 37) [UA]

- Suaedo corniculatae-Puccinellion tenuiflorae MIRKIN ex Golub 1994a all. nov. (: 46) [MN, RU, Yakutiya]

- Alhagienion Golub 1994a suball. nov. (: 37) [RU, Russia South]

- Beckmannio-Puccinellienion bilykianae GoluB 1994a suball. nov. (: 25) [RU, Russia South]

- Geranienion collini GoluB 1994a suball, nov. (: 32) [RU, Russia East, Russia South, Western Siberia]

* Peucedanenion latifolii Golub 1994a suball. nov. (: 25) [art. 2b] [RU, Russia South]

- Tripolio-Phragmitenion GoluB 1994a suball. nov. (: 37) [RU, Russia South]

- Alhagio-Artemisietum santonicae Golub et TCHORBADZE in GOlUB 1994a ass. nov. (: 53) [RU, Russia South]

* Cichorio-Eryngietum plani Golub et Saveljeva in Golub 1994a ass. nov. (: 52) [art. 2b] [RU, Russia South]

- Cirsio esculenti-Hordeetum brevisubulati KarPov, MiRKIN, ONISHChENKo ex Golub 1994a ass. nov. (: 52) [RU, Russia East]

- Cnidio-Poetum angustifoliae Golub 1994a ass. nov. (: 52) [RU, Western Siberia]

- Elytrigio-Artemisietum nitrosae GOLUB 1994a ass. nov. (: 53) [RU, Western Siberia]

- Halerpestetum salsuginosae MiRKIN, NaUmova et SOlomestCh ex Golub 1994a ass. nov. (: 53) [MN]

- Junco gerardii-Beckmannietum eruciformis AGAFONOV el GOLUB in GOLuB 1994a ass. nov. (: 52) [RU, Russia Central]

- Limonio tomentelli-Festucetum valesiacae AGAFONOv et GolUB in GolUB 1994a ass. nov. (: 53) [RU, Russia Central]

- Plantagini cornuti-Festucetum arundinaceae Golub et SAVELJEVA in Golub I994a ass. nov. (: 52) [RU, Russia South]

- Puccinellietum fominii SHEl Yag-SOSONKo et SOlOMAKHa ex Golub 1994a ass. nov. (: 54) [UA]

- Scorzonero parviflorae-Puccinellietum distantis (SOLOMAKHA et SHELYAG-SOSONKo 1984) GOLUB 1994a ass. nov. (: 53) [UA]

- Suaedo-Phragmitetum GOLUB et TCHORBADZE in GOLUB 1994a ass. nov. (: 54) [RU, Russia South]

Asterisco graveolentis-Forskhaletea tenacissimae

O Fredolietum aretioidis (QUÉZEL 1965) QUÉZEL, BARBERO, BENABID et RIVAS-MARTÍNEZ 1994 nom. nov., nom. illeg. (: 569) [art. 30]

Astragalo-Brometea

- Astragaletum caspici BEHÇET 1994b ass. nov. (: 296) [TR]

- Astragaletum kurdici BEHÇET 1994b ass. nov. (: 296, 298) [TR]

- Cousinio iconicae-Astragaletum microcephali TATLI, EYCE et SERIN 1994 ass. nov. (: 283, 285) [TR]

- Digitali ferrugineae-Juniperetum nanae TATLI, EYCE et SERIN 1994 ass. nov. (: 281, 283) [TR]

O Minuartio anatolicae-Onobrychidetum cornutae TATLI, EYCE et SERIN 1994 ass. nov., nom inval. (: 285) [art. 5] [TR]

- Serratulo-Spiraeetum hypericifoliae OCAKVERDI 1994 ass. nov. (: 256) [TR]

O Stipo-Festucetum cratericolae OCAKVERDI 1994 ass. nov., nom. inval. (: 254) [art. 5] [TR]

- Taraxaco-Iridetum spuriae OCAKVERDI 1994 ass. nov. (: 251, 252) [TR]

"Avicennio-Salicornietea australis"

O Avicennio-Salicornietea australis HoFf $1994 \mathrm{cl}$. nov., nom. prov. (: 678) [art. 3b] [NC]

O Avicennio-Salicomietalia australis HOFF 1994 ord. nov., nom. prov. (: 678) [art. 3b] [NC]

O Avicennio-Salicornion australis HoFf 1994 all. nov., nom. prov. (: 678) [art. 3b] [NC] 
O Avicennio-Salicornietum australis HoFf 1994 ass. nov., nom. prov. (: 678) [art. 3b] [NC]

"Baccharetea nivalis"

O Baccharetea nivalis PISANo $1994 \mathrm{cl}$ nov., nom. prov. (: 13) [ar1. 3b] [CL, Chile South]

O Baccharetalia nivalis PISANO 1994 ord. nov., nom. prov. (: 13) [art. 3b] [CL, Chile South]

O Baccharion nivalis PISANO 1994 all. nov., nom. prov. (: 13) [art. 3b] [CL, Chile South]

O Baccharetum nivalis PISANO 1994 ass. nov., nom. prov. $(: 13,20)$ [art. 3b] [CL, Chile South]

"Baeckeo-Codietea incrasatae"

O Baeckeo-Codietea incrasatae HoFf 1994 cl. nov., nom. prov. (: 682) [art. 3b] [NC]

O Baeckeo-Codietalia incrasatae Hoff 1994 ord. nov., nom. prov. (: 682) [art. 3b] [NC]

O Baeckeo-Codion incrasatae HoF 1994 all. nov., nom. prov. (: 682) [art. 3b] [NC]

O Baeckeo-Codietum incrasatae HoFf 1994 ass. nov., nom. prov. (: 682) [art. 3b] [NC]

Betulo-Adenostyletea

- Salicetum glabrae SMETTAN ex EGgEnSBERGER 1994 ass. nov. (: 174) [DE, "Alps"]

$B$ identete a

- Echinochloo cruris-galli-Chenopodietum chenopodioidis PARADIS et LORENZONI 1994 ass. nov. (: 24) [FR, Corse]

- Ranunculo scelerati-Polygonetum hydropiperis GÉHU, KAABECHE el GHARZOUL] 1994b ass. nov. (: 71) [DZ]

Bolboschoenetea maritimi

- Carici-Menyanthetum trifoliatae BEHÇET 1994a ass, nov. (: 237) [TR]

- Triglochino-Bolboschoenetum maritimi BEHÇET 1994a ass. nov. (: 236, 237) [TR]

"Bruguiero-Heritieretea littoralis"

O Bruguiero-Heritieretea littoralis HofF $1994 \mathrm{cl}$. nov., nom. prov. (: 678) [art. 3b] [NC]

O Heritiero-Acanthetalia ilicifolii HoFf 1994 ord. nov., nom. prov. (: 678) [art. 3b] [NC]

O Xylocarpo-Bruguieretalia gymnonhysae HoFF 1994 ord. nov., nom. prov. (: 678) [art. 3b] [NC]

O Heritiero-Acanthion ilicifolii HoFf 1994 all. nov., nom. prov. (: 678) [art. 3b] [NC]

O Xylocarpo-Bruguierion littoralis HoFF 1994 all. nov., nom. prov. (: 678) [art. 3b] [NC]

O Avicennio-Rhizophoretum selalae HofF 1994 ass. nov., nom. prov. (: 678) [art. 3b] [NC]

O Heritiero-Acanthetum ilicifolii HoFF 1994 ass. nov., nom. prov. (: 678) [art. 3b] [NC]

O Xylocarpo-Bruguieretum gymnorhysae HoFF 1994 ass. nov., nom. prov. (: 678) [art. 3b] [NC]

Cakiletea integrifoliae

- Polygono maritimi-Elymetum pycnanthi DíAz GonzÁlez et FERNÁndez PRIETo 1994 ass. nov. (: 463, 464) [ES]

Cakiletea maritimae

- Cakilion euxinae GÉHU, ROMAN et BOULLET 1994 all. nov. (: 86) [RO]

- Cakilo euxinae-Salsoletum kali GÉHU, ROMAN et BOULlET 1994 ass. nov. (: 80) [RO]

- Puccinellio festuciformis-Atriplicetum littoralis GÉHU et BIONDI 1994 ass. nov. (: 75) [FR, Corse]

"Caligono-Aristidetea pungentis"

O Caligono-Aristidetea pungentis GÉHU, KAABECHE et GHARZOULI 1994a cl. nov., nom. inval. (: 375) [art. 5] [DZ]

O Aristidion pungentis GÉHU, KAABECHE et GHARZOUL1 1994a all. nov., nom. inval. (: 375) [art. 8] [DZ]

- Anabasi-Aristidetum pungentis GÉHU, KAABECHE et GHARZOULI 1994a ass. nov. (: 375) [DZ] 
Calluno-Ulicetea

O Calluno-Genistetalia (DuviGnAUD 1944) GÉHU 1994 ord. nov., nom. inval. (: 210) [art. 2b]

O Ulici-Ericenion cinereae (J.-M. et J. GÉHU 1975) GÉHU 1994 suball. nov. et stat. nov., nom. inval. (: 209) [art. 2b]

- Halimio lasianthi-Ulicetum minoris CAPELo, CosTA et LOUSÃ 1994 ass. nov. (: 299) [PT]

- Scillo vernae-Ericetum cinereae BlORET 1994 ass. nov. (: 136) [FR]

Calluno-Vaccinietea

- Lonicero periclymeni-Vaccinietum myrtilli DE FOUCAULT 1994a ass. nov. (: 14) [FR]

Camellietea japonicae

- Dendropanaco-Castanopsion sieboldii KIM, HuKUSIMA et HoshINo 1994 all. nov. (: 70, 71) [KR, "Cheju Is"]

- Cyrtomio fortunei-Quercetum glaucae KIM, HukUSIMA et HosHINO 1994 ass. nov. (: 69) [KR, "Cheju Is"]

- Hosto minoris-Castanopsietum sieboldii KIM, Hukusima et HosHINo 1994 ass. nov. (: 63) [KR, "Cheju Is"]

Caricete a curvulae (see also Juncetea trifidi)

O Arnico montanae-Festucetum paniculatae MiCHALET et PHILIPPE 1994 ass. nov., nom. inval. (: 405) [art. 5] [FR, "Massif Central"]

O Vicio orobi-Festucetum paniculatae MiChalet et PHILIPPE 1994 ass. nov,, nom. inval. (: 410) [art. 5] [FR, "Massif Central"']

Carici-Genistetea lobeli

- Armerio sardoae-Genistetum desoleani VALSECCHI 1994 ass. nov. (: 130) [IT]

- Violo limbarae-Genistetum salzmannii VALSECCHI 1994 ass. nov. (: 129) [IT]

Carpino-Fagetea (see Querco-Fagetea)

"Centrolepidi-Hydrocotyletea alatae"

O Centrolepidi-Hydrocotyletea alatae E. et S. PIGNATTI 1994 cl. nov., nom. inval. (: 61) [art. 3g, 8] [AU, Western Australia]

O Centrolepidi-Hydrocotyletalia alatae E. et S. PIGNATTI 1994 ord. nov., nom. inval. (: 61) [art. 3g, 5] [AU, Western Australia]

O Campylopo-Centrolepidion aristatae E. et S. PIGNATTI 1994 all. nov, nom. inval. (: 58, 61) [art. 5] [AU, Western Australia]

- Centrolepidion polygynae E. et S. PIGNaTTI 1994 all. nov. (: 58,6I) [AU, Western Australia]

- Aphelio-Centrolepidetum aristatae E. et S. PIGNATTI 1994 ass. nov. (: 57) [AU, Western Australia]

- Campylopo-Droseretum ramellosae E. et S. PIGNATTI 1994 ass. nov. (: 57) [AU, Western Australia]

- Campylopo-Polypompholydetum multifidae E. et S. PIGNATTI 1994 ass. nov. (: 57) [AU, Western Australia]

- Centrolepidetum polygynae E. et S. PIGNATri 1994 ass. nov. (: 57) [AU, Western Australia]

Che nopodietea

- Digitario-Amaranthetum crispi ŞTEFAN 1994 ass. nov. (: 76) [RO]

- Erigeronto canadensis-Panicetum miliacei SiTEFAN 1994 ass. nov. (: 75) [RO]

Cisto-Lavanduletea

- Cisto crispi-Pinetum pineae Bartolo, Brullo et Pulvirenti 1994b [1995?] ass. nov. (: 410) [It, Sicilia]

- Cisto salvifolii-Halimietum halimifolii GÉHU et BIONDI 1994 ass. nov. (: 96) [FR, Corse]

- Helichryso microphylli-Genistetum ephedroidis VALSECCHI 1994 ass. nov. (: 130) [IT]

- Rosmarino officinalis-Genistetum sardoae VALSECCHI 1994 ass. nov. (: 131) [IT]

- Ulici parviflorae-Cistetum laurifolii Roselló GIMENO 1994 ass. nov. (: 416) [ES] 
Cisto-Micromerietea

- Micromerio graecae-Ephedretum distachyae Biondi, Ballelli, Allegrez.za, Taffetani et FrancalanCia 1994 ass. nov. (: 55) [IT]

Cleistogenetea squarrosae

- Festucetalia kryloviano-tschujensis KOROLYUK et NAMZALOv 1994 ord. nov. (: 470) [RU, Southern Siberia]

- Festucion tschujensis KorolyuK et NAMZaLov 1994 all. nov. (: 470) [RU, Southem Siberia]

- Carici rupestris-Festucetum krylovianae KOROLYUK et NAMZALOv 1994 ass. nov. (: 470) [RU, Southern Siberia]

- Poo attenuatae-Festucetum tschujensis KorolyuK et NAMZALOv 1994 ass. nov. $(: 470,472)$ [RU, Southern Siberia]

"Colubrinetea asiaticae"

O Colubrinetea asiaticae ОHBA 1994 cl. nov., nom. prov. (: 50) [art. 3b] [MP]

O Colubrinetalia asiaticae OHBA 1994 ord. nov., nom. prov. (: 50) [art. 3b] [MP]

O Colubrinion asiaticae ОНвA 1994 all. nov, nom. prov. (: 50) [art. 3b] [MP]

O Pipturo-Colubrinetum asiaticae OHBA 1994 ass. nov., nom. inval. (: 29) [art. 5] [MP]

Corynephoretea canescentis

- Sedion pyrenaici R. Tuxen ex Rivas-Martínez, Díaz González, Fernández Prieto, Loidi et Penas in Díaz GONZÁLEZ et FERNÁNDEZ PRIETO 1994 all. nov. (: 360) [ES]

Crithmo-Limonietea

- Erodio corsici-Limonion articulati GamisAns et MACCIOLE ex GÉhu et BIONDI 1994 all. nov. (: 79, 88) [FR, Corse]

- Crithmo maritimi-Limonietum contortiramei (R. et R. MOLINIER 1955) GÉHU et BIONDJ 1994 nom. nov. (: 77$)$ [FR, Corse]

- Crithmo maritimi-Limonietum patrimoniensis GÉHU et BıONDI 1994 ass. nov. (: 88) [FR, Corse]

- Limonio corsici-Erodietum corsici GaMisans et MURACCIOle ex GÉHU et BIONDI 1994 ass. nov. (: 85) [FR, Corse]

- Reichardio maritimi-Limonietum articulati MALCUIT ex GÉHU et BIONDI 1994 ass. nov. (: 83) [FR, Corse]

Ctenio-Loudetietea togoensis

O Ctenio-Loudetietea togoensis SINSIN $1994 \mathrm{cl}$. nov., nom. inval. (: 97) [art. 8] [BJ]

O Loudetietalia togoensis SINSIN 1994 ord. nov., nom. inval. (: 98) [art. 8] [BJ]

O Loudetion togoensis SINSIN 1994 all. nov., nom. inval. (: 98) [art. 5] [BJ]

- Aristidetum kerstingii SINSIN 1994 ass. nov. (: 98, 103) [BJ]

- Vernonietum perrottetii SINSIN 1994 ass. nov. $(: 98,103)$ [BJ]

Cytisetea scopario-striati

- Calicotomo infestae-Adenocarpetum commutati BarTOLO, BRUllo et PulviRENTI 1994a [1995?] ass. nov. (: 404) [IT, Sicilia]

- Ulici europaei-Cytisetum striati RIvAs-MARTínEZ ex DÍAZ GONZÁleZ et FERnÁNDEZ PRIETo 1994 ass. nov. (: 327 ) [ES]

Deschampsio-Asteretea

O Fragarietalia chiloensis PISANo 1994 ord. nov., nom. prov. (: 12) [art. 3b] [CL, Chile South]

O Fragarion chiloensis PISANO 1994 all. nov., nom. prov. (: 12) [art. 3b] [CL, Chile South]

O Fragarietum chiloensis PISANO 1994 ass. nov., nom. prov. $(: 12,16)$ [art. 3b] [CL, Chile South] 
"Diheteropogono amplectentis-Schizachyrion sanguinei"

O Diheteropogono amplectentis-Schizachyrion sanguinei BEZUIDENHOUT, BREDENKAMP et THERON 1994a all. nov., nom. inval. (: 79) [art. 5, 17] [ZA]

- Hyparrhenio hirtae-Eragrostietum planae BEZUIDENHOUT, BREDENKAMP et THERON 1994a ass. nov. (: 80) [ZA]

- Monocymbio ceresiformis-Schizachyrietum sanguinei BEZUIDENHOUT, BREDENKAMP et THERON 1994a ass. nov. (: 80) [ZA]

- Uryletro agropyroidis-Schizachirietum sanguinei BEZUIDENHOUT, BREDENKAMP et THERON 1994a ass. nov. (: 80) [ZA]

"Diplachnofuscae-Stipagrostietea uniglumis"

O Diplachno fuscae-Stipagrostietea uniglumis BEZUIDENHOUT, BREDENKAMP et THERON 1994d cl. nov. nom. inval. (: 9) [art. 2b, 5,8] [ZA, Transvaal]

"Elaeocarpetea jogae"

O Elaeocarpetea jogae OHBA 1994 cl. nov., nom. inval. (: 52) [art. 8] [MP]

O Aglaio-Elaeocarpetalia OHBA 1994 ord. nov., nom. inval. (: 52) [art. 8] [MP]

O Aglaio-Guamion marianae OHBA 1994 all. nov., nom. inval. (: 52) [art. 8] [MP]

O Aglaio-Elaeocarpetum jogae OHBA 1994 ass. nov., nom. inval. (: 19) [art. 5] [MP]

"Elaeocarpo-Pandanetea macrocarpi"

O Elaeocarpo-Pandanetea macrocarpi Hoff 1994 cl. nov., nom. prov. (: 680) [art. 3b] [NC]

O Elaeocarpo-Pandanetalia macrocarpi HOFF 1994 ord. nov., nom. prov. (: 680) [art. 3b] [NC]

O Elaeocarpo-Pandanion macrocarpi Hoff 1994 all. nov., nom. prov. (: 680) [art. 3b] [NC]

O Elaeocarpo-Pandanetum macrocarpi HoFF 1994 ass. nov, nom. prov. (: 680) [art. 3b] [NC]

"Elaeocarpo-Rhoetea taitensis"

O Elaeocarpo-Rhoetea taitensis HOFF $1994 \mathrm{cl}$. nov., nom. prov. (: 680) [art. 3b] [NC]

O Elaeocarpo-Rhoetalia taitensis HoFf 1994 ord. nov., nom. prov. (: 680) [art. 3b] [NC]

O Elaeocarpo-Rhoion taitensis HOFF 1994 all. nov., nom. prov. (: 680) [art. 3b] [NC]

O Elaeocarpo-Rhoetum taitensis HOFF 1994 ass. nov., nom. prov. (: 680) [art. 3b] [NC]

Elyno-Seslerietea

- Caricenion austroalpinae Poldinj et Feoli ChiAPElla in FEOLI Chiapella et POLdiNı 1994 suball. nov. (: 16)

- Ranunculenion hybridi PoldiNi et FeOLi ChiAPELla in FeOli Chiapella et Poldini 1994 suball. nov. (: 16, 120) [IT]

O Aquilegio-Anemonetum narcissiflorae TOMASELLI 1994 ass. nov., nom. inval. (: 43, 46) [art. 3g] [IT]

- Carici ornithopodae-Seslerietum albicantis POLDINI et FEOLI CHIAPELLA in FEOLI ChIaPELLA et POLDINI 1994 ass. nov. (: 65) [IT]

- Globulario cordifoliae-Caricetum humilis BERNÁTOvÁ et UhLír̃ová 1994 ass. nov. (: 2) [SK]

- Pediculari comosae-Caricetum sempervirentis DÍAZ GONZÁLEZ el FERNÁNDEZ PRIETO 1994 ass. nov. (: 338) [ES]

- Ranunculo hybridi-Caricetum sempervirentis POLdINI et FEOLI CHIAPELLA in FEOLI ChIAPELLA et POLDINI 1994 ass. nov. (: 76) [IT]

O Saxifrago speciosae-Silenetum cenisiae PETRiCCIONE 1994 ass. nov., nom. inval. (: 197, 198) [art. 5, 7] [IT]

"Eragrostio planae-Hyparrhenietea hirtae"

O Eragrostio planae-Hyparrhenietea hirtae BEZUIDENHOUT, BREDENKAMP et THERON 1994d cl. nov. nom. inval. (: 15) [art. 2b, 5, 8] [ZA, Transvaal] 
"Eragrostio racemosae-Trachypogonetea spicati"

O Eragrostio racemosae-Trachypogonetea spicati BEZUIDENHOUT, BREDENKAMP et THERON 1994d cl. nov. nom. inval. (: 9) [art. 2b, 5, 8] [ZA. Transvaal]

Erico-Pinetea (see Querco-Fagetea)

Euphorbio-Ammophiletea arundinaceae

* Medicagini-Ammophilenion arundinaceae (RIVAS-MARTíneZ et GÉHU 1980) GÉHU et BIONDI 1994 nom. nov., nom. superfl. (: 23, 135, 143) [art. 29]

- Inulo crithmoidis-Elymetum farcti PIAzZa et PARADIs 1994 ass. nov. (: 68) [FR, Corse]

* Sporoboletum arenarii ARÈNEs ex GÉHU et BIONDI 1994 ass. nov., nom. superfl. (: 135, 142) [art. (25) 29] [FR, Corse]

Festuco-Brome te a (see also Phlomidi-Brachypodietea)

O Galio campanulatae-Poion versicoloris KuKovitsa, Movchan, SOlomaKha et SHELYAG-SOSONKo 1994 all. nov., nom. inval. (: 37) [art. 5, 17] [UA]

- Centaureenion dichroanthae (PIGNatTI 1953) POldini et Feoli Chiapella in Feoli Chiapella et POLdini 1994 suball. nov. et stat. nov. (: 11,118$)$

$\Delta$ Hypochoeridenion maculatae (HoRvatí 1973) POLdiNt et Feoli Chiapella in FeOl Chiapella et POLDinj 1994 suball. nov. et stat. nov. (: 12,119$)$

$\Delta$ Scononerenion villosae Poldini et Feoli ChIAPElla in Feol. ChIAPElla et POLdiNi 1994 suball. nov. (: 12)

O Acini arvensis-Elytrigietum intermediae KuKOVITSA, MOVCHAN, SOLOMAKHA et SHELYAG-SOSONKO 1994 ass. nov. (: 47) [art. 5, 16] [UA]

O Adonido vernalis-Stipetum tirsae Kukovitsa, Movchan, SolomakHa et Shelyag-Sosonko 1994 ass. nov. (: 37 ) [art. 5, 16] [UA]

O Alchemilletum plicatissimae KLEIN et LACOSTE 1994 ass. nov., nom. inval. (: 408) [art. 5, 16] [IR]

O Artemisio albae-Thesietum divaricati BRAQUE et LOISEAU 1994 ass. nov., nom. inval. (: 44) [art. 5] [FR]

- Avenulo praeustae-Brometum erecti POLdiNI et Feoli ChIAPElla in Feoli ChIAPElla et POLdiNi 1994 ass. nov. (: 57) $[$ IT]

- Bromo erecti-Onobrychidetum montanae DELPECH 1994 ass. nov. (: 356) [FR, "Alps"]

- Bupleuro-Brometum condensati POLDINI et FEOLI CHIAPElla in FEOLI ChIAPELla et POLdiNi 1994 ass. nov. (: 28) [IT]

O Carici flaccae-Tetragonolobetum maritimi GŁAZEK et EUSZCZYNSKA 1994 ass. nov., nom. inval. (: 281, 282) [art. 5] [PL]

O Carici humilis-Arctostaphyletum uvae-ursi PETRICCIONE 1994 ass. nov., nom. inval. (: 197, 199) [art. 5, 7] [IT]

O Centaureo approximatae-Knautietum arvensis BRAQUE et LOISEAU 1994 ass. nov, nom. inval. (: 144) [art. 5] [FR]

$\Delta$ Chamaecytiso hirsuti-Chrysopogonetum grylli PIGNATTI ex POLDINI et FEOLi ChIAPELla in FeOLI ChIAPELla et POLDINI 1994 ass. nov. (: 38) [IT]

- Euphrasio tricuspidatae-Seslerietum variae PEDRoTTI et MINGHETTI in PEDROTTI 1994 ass. nov. (: 39) [IT, “Alps"]

- Gladiolo palustris-Molinietum arundinaceae POLdiNi et FEOLI ChIAPELLA in FEOLI CHIAPELLA et POLDINI 1994 ass. nov. (: 50) [IT]

O Gypsophilo fastigiatae-Teucrietum chamaedrys KuKovitSA, MOvchan, SOLOMAKHA et SHELYAG-SOSONKO 1994 ass. nov. (: 37) [art. 5, 16] [UA]

O Helianthemo apennini-Brometum erecti BRAQUE et LOISEAU 1994 ass. nov., nom. inval. (: 137) [art. 5] [FR]

O Hyssopo decumbentis-Arenarietum controversae BRAQUE et LOISEAU 1994 ass. nov., nom. inval. (: 34) [art. 5] [FR]

O Jurineo calcareae-Stipetum capillatae KukovitSA, MovCHAN, SOlOMAKHA et Shelyag-Sosonko 1994 ass. nov. (: 46) [art. 5, 16] [UA]

O Lembotropo nigricantis-Potentilletum arenariae KukovitSA, Movchan, SOLOMAKHA et ShElYaG-SOSONKO 1994 ass. nov. (: 46) [art. 5, 16] [UA]

O Leucanthemo graminifolii-Seslerietum albicantis BRAQUE et LOISEAU 1994 ass. nov., nom. inval. (: 53) [art. 5] [FR]

- Onobrychido arenariae-Brometum Poldini et Feoli ChIAPElla in Feoli ChIAPElla et PoldiNi 1994 ass. nov. (: 45) [IT]

O Ranunculo zapalowiczii-Helictotrichetum desertori KukOvitsa, MOVCHan, SOLOMAKHA et SHELYAG-SOSONKO 1994 ass. nov. (: 37) [art. 5, 16] [UA]

O Sanguisorbo muricatae-Caricetum hallerianae BRAQUE et LOISEAU 1994 ass. nov., nom. inval. (: 72) [art. 5] [FR]

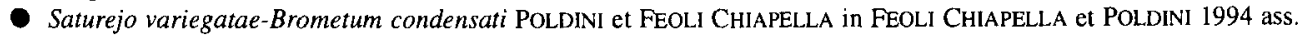
nov. (: 22) [IT] 
Schoeno nigricantis-Chrysopogonetum grylli PIGNATTI ex FEOLI CHIAPELLA et POLDINI 1994 ass. nov. (: 34) [IT]

O Violo rupestris-Koelerietum pyramidatae BRAQUE et LOISEAU 1994 ass. nov, nom. inval. (: 121) [art. 5] [FR]

"Festuco hystricis-Ononidetea striatae" (see also Ononido-Rosmarinetea)

Festuco-Seslerietea

O Gnaphalio diminuti-Festucetum circummediterraneae PETRICCIONE 1994 ass. nov., nom. inval. (: 197, 199) [art. 5 , 7] [IT]

Filipendulo-Convolvuletea

O Lythro salicariae-Filipenduletalia (PASSARGE 1988) JULVE et GlLLET 1994 ord. nov. et stat. nov., nom. inval. (: 58, 59) [art. 2b]

O Stachyo palustris-Cirsion oleracei JULVE et GILLET 1994 all. nov., nom. inval. (: 59) [art. 8]

O Filipendulo niveae-Caricetum cespitosae JULvE et GlLLET 1994 ass. nov., nom. inval. (: 58) [art. 3f] [PL]

O Junco acutiflori-Brometum racemosi KLAPP eX DE FOUCAULT 1994b ass. nov., nom. inval. (: 22) [art. 5] [FR]

Galio-Urticetea

- Violo-Stellarion holosteae PASSARGE 1994b all, nov. (: 185) [DE]

- Glechomo-Cucubaletum bacciferi GÉHU et BOURNIQUE 1994 ass. nov. (: 100) [FR]

- Veronico-Stellarietum holosteae PASSARGE 1994b ass. nov. (: 181) [DE]

"Gardenio-Acacietea spirorbis"

O Gardenio-Acacietea spirorbis HoFf 1994 cl. nov., nom. prov. (: 682) [art. 3b] [NC]

O Gardenio-Acacietalia spirorbis HoFf 1994 ord. nov., nom. prov. (: 682) [art. 3b] [NC]

O Gardenio-Acacion spirorbis HOFF 1994 all. nov., nom. prov. (: 682) [art. 3b] [NC]

O Bauerello-Melaleucetum quinquenerviae HoFf 1994 ass. nov., nom. prov. (: 682) [art. 3b] [NC]

O Dodonaeo-Acacietum spirorbis HoFf 1994 ass. nov., nom. prov. (: 682) [art. 3b] [NC]

"Grewio flavae-Acacietea karroo"

O Grewio flavae-Acacietea karroo BEZUIDENHOUT, BREDENKAMP et THERON 1994d cl. nov., nom. inval. (: 14) [art. 2b, 5, 8] [ZA, Transvaal]

O Grewio flavae-Rhoion pyroidis BEZUIDENHOUT, BREDENKAMP et THERON 1994b all. nov,, nom. inval. (: 158) [art. 5, 17] [ZA, Transvaal]

O Protasparago africani-Acacion karroo BEZUIDENHOUT, BREDENKAMP et THERON 1994d all. nov., nom. inval. (: 15) [art. 2b, 5,8] [ZA, Transvaal]

- Rhoo lanceae-Acacietum eriolobae BEZUIDENHOUT, BREDENKAMP et THERON 1994b ass. nov. (: 158) [ZA, Transvaal]

- Zizipho mucronatae-Acacietum karroo BEZUIDENHOUT, BREDENKAMP el THERON 1994b ass. nov. (: 158) [ZA, Transvaal]

"Guettardo-Pandanetea tectorum"

O Guettardo-Pandanetea tectorum HoFf $1994 \mathrm{cl}$. nov., nom. prov. (: 679) [art. 3b] [NC]

O Guettardo-Pandanetalia tectorum HoFf 1994 ord. nov., nom. prov. (: 679) [art. 3b] [NC]

O Euphorbio-Lysimachion mauritianae HOFF 1994 all. nov., nom. prov. (: 679) [art. 3b] [NC]

O Excoecario-Pemphision acidulae HoFf 1994 all. nov., nom. prov. (: 679) [art. 3b] [NC]

O Lepturo-Excoecarion agallochae HoFf 1994 alt. nov., nom. prov. (: 679) [art. 3b] [NC]

O Pandano-Araucarion columnaris HoFf 1994 all. nov., nom. prov. (: 679) [art. 3b] [NC]

O Asplenio-Hoyetum neocaledonicae HOFF 1994 ass. nov., nom. prov. (: 679) [art. 3b] [NC]

O Colubrino-Vitietum trifoliatae HoFf 1994 ass. nov., nom. prov. (: 679) [art. 3b] [NC]

O Euphorbio-Lysimachietum mauritianae HoFF 1994 ass. nov., nom. prov. (: 679) [art. 3b] [NC] 
O Excoecario-Pemphisetum acidulae HoFf 1994 ass. nov., nom. prov. (: 679) [art. 3b] [NC]

O Lepturo-Pandanetum tectorum HoFf 1994 ass. nov., nom. prov. (: 679) [art. 3b] [NC]

O Leucaeno-Acacietum simplicis Hof 1994 ass. nov., nom. prov. (: 679) [art. 3b] [NC]

O Pandano-Araucarietum columnaris HoFf 1994 ass. nov., nom. prov. (: 679) [art. 3b] [NC]

O Phymatosoro-Araucarietum columnaris HOFF 1994 ass. nov., nom. prov. (: 679) [art. 3b] [NC]

O Phymatosoro-Excoecarietum agallochae HoFf 1994 ass. nov., nom. prov. (: 679) [art. 3b] [NC]

O Scaevolo-Cocetum nuciferae HoFf 1994 ass. nov, nom. prov. (: 679) [art. 3b] [NC]

O Sporobolo-Acacietum simplicis Hoff 1994 ass. nov., nom. prov. (: 679) [art. 3b] [NC]

Helichryso-Crucianelletea

- Helichrysetalia italici GÉHU et BIONDI 1994 ord. nov. (: 143) [FR, Corse]

- Euphorbion pithyusae GÉHU et BIONDI 1994 all nov. (: 143) [FR, Corse]

- Ononido ramosissimae-Polycarpion niveae BIONDI, ALLEgrezZA, TAFFETANI et WILDPRET DE LA TORRE 1994 all nov. (: 110) [ES]

- Helichryso microphylli-Asteriscetum maritimi GAMISANS ex GÉHU et BIONDI 1994 ass. nov. (: 106) [FR, Corse]

- Launaeo arborescentis-Ononidetum ramosissimae BIONDI, AllegreZZA, TAFFETANI et WILDPRET DE LA TORRE 1994 ass. nov. (: 110) [ES]

- Scrophulario ramosissimae-Genistetum salzmannii MALCUIT ex GÉHU et BIONDI 1994 ass. nov. (: 96) [FR, Corse]

Hibisco-Pandanetea

O Hibiscetum tiliacei OHBA 1994 ass. nov., nom. inval. (: 22) [art. 5] [MP]

Honckenyo-Elymetea

- Artemisio tscherniaevianae-Centaureetum odessanae GÉHU, ROMAN et BOULLET 1994 ass. nov. (: 82) [RO]

Hyparhenietea

O Andropogonetalia bisquamulati SINSIN 1994 ord. nov., nom. inval. (: 97) [art. 8] [BJ]

O Hyparhenio-Andropogonion tectorum SINSIN 1994 all. nov, nom. inval. (: 97) [art. 5] [BJ]

O Schizachyrio-Loxoderion ledermannii SINSIN 1994 all. nov., nom. inval. (: 97) [art. 5] [BJ]

- Aspilio-Anadelphietum afzelianae SINSIN 1994 ass. nov. $(: 97,101)[\mathrm{BJ}]$

- Loudetietum flavidae SINSIN 1994 ass. nov. (: 97, 102) [BJ]

- Loxoderetum ledermannii SINSIN 1994 ass. nov. (: 97, 102) [BJ]

- Pennisetum uniseti SINSIN 1994 ass. nov. (: 97, 101) [BJ]

Isoeto-Nanojuncetea

- Atriplici prostratae-Crypsietum aculeatae PARADIS el LORENZONI 1994 ass. nov. (: 19) [FR, Corse]

- Chenopodio chenopodioidis-Crypsiettm aculeatae (PARADIS 1992) PARADIS et LORENZONI 1994 ass. nov. (: 21) [FR, Corse]

- Chenopodio chenopodioidis-Crypsietum schoenoidis (PARADIS 1992) PARADIS et LoRENZONI 1994 ass. nov. (: 22) [FR, Corse]

- Crypsio aculeatae-Cressetum creticae GÉHU, KAABECHE et GHARZOULu 1994c ass. nov. (: 302) [DZ]

- Echinochloo cruris-galli-Crypsietum schoenoidis (PARADIS 1992) PARADIS et LORENZONI 1994 ass. nov. (: 22) [FR, Corse]

O Isoeto-Juncetum pygmaei GÉHU, KAABECHE et GHARZOULI 1994c ass. nov., nom. inval. (: 307) [art. 2b] [DZ]

- Laurentio bicoloris-Fimbristylidetum squarrosae GÉHU, KAABECHE et GHARZOULI 1994c ass. nov. (: 304) (DZ]

- Laurentio michelii-Isolepidetum cernui GEHU, KAABECHE et GHARZOULI 1994c ass. nov. (: 304) [DZ]

- Polygono monspeliensis-Crypsietum aculeatae PARADIS et LORENZONI 1994 ass. nov. (: 21) [FR, Corse]

- Samolo valerandi-Crypsietum aculeatae PARAdIS et LoRENzONi 1994 ass. nov. (: 21) [FR, Corse]

- Scirpo cernui-cyperetum flavescentis ROSELLo GIMENO 1994 ass. nov. (: 378) [ES]

- Scirpo setacei-Juncetum bufonii (VIGO 1968) ROSELLó GIMENO 1994 nom. nov. (: 378) [ES]

Juncetea maritimi

$\triangle$ Artemisio santonici-Juncetum littoralis (POPESCU et SANDA 1976) GÉHU, Roman et BoUllet 1994 ass. nov. (: 76) [RO] 
- Astero pannonici-Juncetum gerardii GÉHU, ROMAN et BOULLET 1994 ass, nov. (: 76) [RO]

- Astero pannonici-Puccinellietum distantis GÉHU, ROMAN et BOULlET 1994 ass. nov. (: 76) [RO]

- Halimiono pedunculatae-Aeluropetum GÉHU, ROMAN et BOULLET 1994 ass. nov. (: 76) [RO]

- Halimiono pedunculatae-Juncetum maritimi GÉHU, ROMAN et BOULLET 1994 ass. nov. (: 76) [RO]

$\Delta$ Limonio gmelinii-Artemisietum santonici (TzOPA 1939) GÉHU, ROMAN et Boullet 1994 nom. nov. (: 76) [RO]

- Limonio narbonensis-Caricetum extensae GÉHU et BIONDI 1994 ass. nov. (: 72) [FR, Corse]

- Limonio narbonensis-Juncetum gerardii GÉHU et BIONDI 1994 ass. nov. (: 73) [FR, Corse]

- Puccinellio convolutae-Halimionetum verruciferae GÉHU, ROMAN et BOULLET 1994 ass. nov. (: 76) [RO]

- Puccinellio convolutae-Limonietum danubialis GÉHU, ROMAN et BOULLET 1994 ass. nov. (: 74, 76) [RO]

Juncetea trifidi (see also Caricetea curvulae)

- Sileno exscapae-Trifolietum alpini TOMASELlI et ROSSI 1994 ass. nov. (: 54) [IT]

Ko elerio-Corynephorete a (see also Sedo-Scleranthetea)

$\Delta$ Centaureo rhenanae-Festucetum ovinae KRAUSCH ex KOWARIK et LANGER 1994 ass. nov. [?] (: 13, 15, 35) [DE] [sub: Centaureo rhenanae-Festucetum ovinae KRAUSCH $1959 \mathrm{em}$. KOWARIK et LANGER]

"Lastreopsio-Cupaniopsietea azanthae"

O Lastreopsio-Cupaniopsietea azanthae HoFF 1994 cl. nov., nom. prov. (: 680) [art. 3b] [NC]

O Cupaniopsio-Cryptocaryetalia oubatche HoFF 1994 ord. nov., nom. prov. (: 680) [art. 3b] [NC]

O Planchonello-Hedycaryetalia cupulatae HoFf 1994 ord. nov., nom. prov. (: 681) [art. 3b] [NC]

O Cupaniopsio-Cryptocaryion oubatche HoFF 1994 all. nov., nom. prov. (: 680) [art. 3b] [NC]

O Planchonello-Hedycaryion cupulatae HoFf 1994 all. nov., nom. prov. (: 681) [art. 3b] [NC]

O Dysoxylo-Tapeinospermetum oblongae HoFf 1994 ass. nov., nom. prov. (: 681) [art. 3b] [NC]

O Lastreopsio-Cryptocaryetum macrocarpae HoFf 1994 ass. nov., nom. prov. (: 681) [art. 3b] [NC]

O Planchonello-Hedycaryetum cupulatae HoFf 1994 ass. nov., nom. prov, (: 681) [art. 3b] [NC]

Lem netea

O Azollo filiculoidis-Lemnetum minusculae FELZINES et LOISEAU 1991 nom. invers., nom. illeg. in WOLFF, DIEKJOBST et SCHWARZER 1994 (: 349) [art. 42]

O Spirodelo polyrrhizae-Salvinietum natantis SLAVNIĆ 1956 nom. invers., nom. illeg. in WOLFF, DIEKJOBST et SCHWARZER 1994 (: 352) [art. 42]

- Wolffio-Ricciocarpetum natantis GÉHU, KAABECHE et GHARZOULI 1994c ass. nov. (: 303) [DZ]

"Lepturo-Atriplicetea jubatae"

O Lepturo-Atriplicetea jubatae HoFF 1994 cl. nov., nom. prov. (; 678) [art. 3b] [NC]

O Lepturo-Atriplicetalia jubatae HoFf 1994 ord. nov., nom. prov. (: 678) [art. 3b] [NC]

O Cakilo-Spinificion hirsutae HOFF 1994 all. nov., nom. prov. (: 678) [art. 3b] [NC]

O Salsolo-Atriplicion jubatae HofF 1994 all. nov., nom. prov. (: 678) [art. 3b] [NC]

O Scaevolo-Surianion maritimae HoFF 1994 all. nov., nom. prov. (: 678) [art. 3b] [NC]

O Cakilo-Spinificetum hirsutae HoFf 1994 ass. nov., nom. prov. (: 678) [art. 3b] [NC]

O Salsolo-Atriplicetum jubatae HoFF 1994 ass. nov., nom. prov. (: 678) [art. 3b] [NC]

O Scaevolo-Surianetum maritimae HoFf 1994 ass. nov., nom. prov. (: 678) [art. 3b] [NC]

O Sporobolo-Sesuvietum portulacastri Hoff 1994 ass. nov., nom. prov. (: 678) [art. 3b] [NC]

"Lindsaeo-Cupaniopsietea oedipodae"

O Lindsaeo-Cupaniopsietea oedipodae HoFF 1994 cl. nov., nom. prov. (: 681) [art. 3b] [NC]

O Alstonio-Cleidietalia lasiophyllae HoFf 1994 ord. nov., nom. prov. (: 681) [art. 3b] [NC]

O Psychotrio-Kibaropsietalia caledonicae HoF 1994 ord. nov., nom. prov. (: 681) [art. 3b] [NC]

O Agathido-Araucarion schmidii HofF 1994 all. nov., nom. prov. (: 681) [art. 3b] [NC]

O Alstonio-Cleidiion lasiophyllae HoFF 1994 all. nov., nom. prov. (: 681) [art. 3b] [NC] 
O Phyllantho-Psychotrion balansae HoFF 1994 all. nov., nom. prov. (: 681) [art. 3b] [NC]

O Agathido-Araucarietum schmidii HoFf 1994 ass. nov., nom. prov. (: 681) [art. 3b] [NC]

O Alstonio-Cleidietum lasiophyllae HoFf 1994 ass. nov., nom. prov. (: 681) [art. 3b] [NC)

O Phyllantho-Psychotrietum balansae HoFF 1994 ass. nov., nom. prov. (: 681) [art. 3b] [NC]

"Loudetio simplicis-Schizachyrietalia sanguinei"

O Loudetio simplicis-Schizachyrietalia sanguinei BEZUIDENHOUT, BREDENKAMP et THERON 1994c ord. nov., nom. inval. (: 221) [art. 2b, 5] [ZA, Transvaal]

O Schizachyrio sanguinei-Pogonarthrion squarrosae BEZUIDENHOUT, BREDENKAMP et THERON 1994c all. nov., nom. inval. (: 222) [art. 5] [ZA, Transvaal]

O Schizachyrio sanguinei-Vanguerion infaustae BEZUIDENHOUT, BREDENKAMP et THERON 1994c all. nov., nom. inval. (: 221) [art. 5] [ZA, Transvaal]

- Alloteropsido semialatae-Pogonarthrietum squarrosae BEZUIDENHOUT, BREDENKAMP et THERON $1994 \mathrm{c}$ ass. nov. (: 222) [ZA, Transvaal]

- Aristido stipitatae-Pogonarthrietum squarrosae BeZUIDENHOUT, BREDENKAMP et THERON 1994c ass. nov. (: 222) [ZA, Transvaal]

- Pavetto zeyheri-Vanguerietum infaustae BEZUIDENHOUT, BREDENKAMP et THERON 1994c ass. nov. (: 221) [ZA. Transvaal]

- Zanthoxylo capensis-Vanguerietum infaustae BEZUIDENHOUT, BREDENKAMP et THERON 1994c ass. nov. (: 221) [ZA, Transvaal]

"Lupino-Calamagrostion tolucensis"

O Lupino-Calamagrostion tolucensis AlMEIDA, CleEF, HeRRERA, VelAZQUeZ et LUNA 1994 all. nov., nom. prov. (: 404) [art. 3b] [MX, Mexico Central]

- Lupino montani-Festucetum tolucensis AlMEIDA, CleEF, HeRRERA, Velazquez et LuNA 1994 ass. nov. (: 409) [MX, Mexico Central]

- Penstemono gentianoidis-Lupinetum montani AlmEIdA, Cleef, Herrera, VelazQuez et LunA 1994 ass. nov. (: 410) [MX, Mexico Central]

- Senecioni procumbentis-Calamagrostietum tolucensis AlmeIda, CleEF, Herrera, VelazQuez et LuNA 1994 ass. nov. (: 404) [MX, Mexico Central]

Lycopodietea cernui

O Gleichenio-Dicranopteridetalia linearis HoFF 1994 ord. nov., nom. prov. (: 682) [art. 3b] [NC]

O Baeckeo-Pteridion esculentis HoFf 1994 all. nov., nom. prov. (: 682) [art. 3b] [NC]

O Melaleuco-Gleichenion dicarpae HoFf 1994 all. nov., nom. prov. (: 682) [art. 3b] [NC]

O Nephrolepido-Hibiscion tiliacei HoFf 1994 all. nov., nom. prov. (: 682) [art. 3b] [NC]

O Baeckeo-Pteridietum esculentis HoFF 1994 ass. nov., nom. prov. (: 682) [art. 3b] [NC]

O Costulario-Gleichenietum flabellatae HoF 1994 ass. nov., nom. prov. (: 682) [art. 3b] [NC]

O Dicranopterido-Cymbopogonetum refracti HoFF 1994 ass. nov., nom. prov. (: 682) [art. 3b] [NC]

O Gleichenio-Baeckeetum ericoidis HoFf 1994 ass. nov., nom. prov. (: 682) [art. 3b] [NC]

O Melaleuco-Chorizandretum cymbariae Hoff 1994 ass. nov., nom. prov. (: 682) [art. 3b] [NC]

O Melaleuco-Gahnietum novocaledonensis HofF 1994 ass. nov., nom. prov. (: 682) [art. 3b] [NC]

Lygeo sparti-Stipetea tenacis $\mathrm{i}$ mae (see also Phlomidi lychnitidis-Brachypodietea retusi)

O Association à Fagonia fruticans et Ferula vesceritensis KAABECHE et GHARZOULI 1994 ass. nov., nom. inval. (: 599) [art. 3h] [DZ]

- Festuco scariosae-Helictotrichetum arundani ASENSI, DIEZ-GARRETAS et MARTín OSORIO 1994 ass. nov. (: 5) [ES]

* Helictotricho bromoidis-Stipetum pauneroanae (VIGO 1968) RoSElló GiMENO 1994 nom. nov., nom., superfl. (: 415) [art. 29]

Malacanthion alnifoliae

- Malacanthion alnifoliae VANDEN BERGHEN 1994 all. nov. (: 20) [SN]

- Malacantho-Cnestidetum ferruginae VANDEN BERGHEN 1994 ass. nov. (: 20, ) [SN] 
Malacantho-Zanthoxyletum zanthoxyloidis VANDEN BERGHEN 1994 ass. nov. (: 20) [SN]

"Melampyro-Holcetea mollis"

O Melampyro-Holcetea mollis KLAUCK ex PASSARGE 1994c cl. nov., nom. inval. (: 107, 108) [art. 8]

O Holco mollis-Pteridienion PASSARge 1994c suball. nov., nom. inval. (: 107) [art. 8]

- Potentillo-Holcenion mollis PASSARge 1994c suball. nov. (: 107)

O Veronico-Hieracienion laevigatae PASSARGE $1994 \mathrm{c}$ suball. nov., nom. inval. (: 108) [art. 3g]

- Avenello-Luzuletum sylvaticae BRAUN-BLANQUET et TÜXEN ex PASSARGE 1994c ass. nov. (: 101) [DE, FR, IE, GB]

- Holco mollis-Equisetetum sylvatici PASSARGE 1994c ass. nov. (: 99, 100) [DE]

- Holco mollis-Melampyretum sylvatici PASSARGE 1994c ass, nov. (: 89) [DE]

- Holco mollis-Pteridietum aquilini PASSARGE 1994c ass. nov. (: 101, 102) [DE]

- Melampyro-Hieracietum sabaudi KLAUCK ex PASSARGE 1994c ass. nov. (: 91) [DE]

- Osmundo-Blechnetum spicantis CLÉMENT et TOUFFET ex PASSARGE 1994c ass. nov. (: 105) [FR]

$\triangle$ Poo angustifoliae-Melampyretum pratensis (WOJTERSKI et al. 1976) BRZEG (1988) PASSARGE 1994c nom. nov. (: 88) [PL]

- Trientali europaeae-Pteridietum aquilini PASSARGE 1994c ass. nov. (: 104) [DE]

- Veronico chamaedryos-Lathyretum linifolii PASSARGE 1994c ass. nov. (: 93, 95) [DE]

"Miscanthetea floriduli"

O Miscanthetea floriduli OHBA $1994 \mathrm{cl}$. nov., nom. inval. (: 52) [art. 8] [MP]

O Miscanthetalia floriduli OHBA 1994 ord. nov., nom. inval. (: 52) [art. 8] [MP]

O Miscanthetion floriduli OHBA 1994 all. nov., nom. inval. (: 52) [art. 8] [MP]

O Melastomo-Lycopodietum cernui OHBA 1994 ass. nov., nom. inval. (: 40) [art. 5] [MP]

O Miscanthetum floriduli OHBA 1994 ass. nov., nom. inval. $(: 37,39)$ [art. 5] [MP]

Molinio-Arrhenatheretea

- Prunello pyrenaicae-Arrhenatherenion bulbosi Díaz GonzÁlez el FERnández PrIETo 1994 suball. nov. (: 371 , 372) $[\mathrm{ES}]$

- Agrostietum gigantei SANDa, POPESCu et Serbanescu 1994 ass. nov. (: 15) [RO]

$\triangle$ Agrostio prorepentis-Ranunculetum repentis (KNAPP 1946) JULVE et GILLET 1994 (: 62) [PL]

- Alopecuretum arundinaceae BEHÇET 1994a ass. nov. (: 239) [TR]

- Cypero-Caricetum crupinae TUXEN ex DÍAZ GONZÁLEZ et FERNÁNDEZ PRIETo 1994 ass. nov. (: 386, 387) [ES]

- Dorycnio recti-Juncetum maritimi RoselLó GIMENo 1994 ass. nov. (: 385) [ES]

- Galio maritimi-Agrostietum castellanae ROSELLó GIMENO 1994 ass. nov. (: 386) [ES]

- Hyperico maculati-Juncetum effusi JULvE et GILLET 1994 (: 61) [PL]

- Loto pedunculati-Juncetum conglomerati HERRERA et FERnÁNDEZ PRIETO in DíAz GonZÁLEZ et FERNÁNDEZ PRIETO 1994 ass. nov. (: 375,376$)$ [ES]

- Oenantho crocatae-Filipenduletum ulmariae DÍAz GonZÁlez et FERnÁNDEZ PRIETo 1994 ass. nov. (: 380) [ES]

- Paspaletum dilatato-distichi Herrera et Fernández Prieto in Díaz González et Fernández Prieto 1994 ass. nov. (: 387, 388) [ES]

* Senecioni laderoi-Filipenduletum ulmariae Rivas-Martínez, Díaz González, Fernández Prieto, Loidi et Peñas 1984 corr. Díaz GonzÁl.eZ et Fernández PRIETo 1994 (: 379) [art. 43] [ES]

Molinto-Juncetea

- Deschampsio-Elymetum repentis OCAKVERDI 1994 ass. nov. $(: 256,258)$ [TR]

- Taraxaco-Iridetum musulmanicae BEHÇET 1994c ass. nov. (: 308, 309) [TR]

Montio-Cardaminetea

- Epilobio nutantis-Montion ZECHMEISTER in ZECHMEISTER et MUCINA 1994 all. nov. (: 400)

- Adiantenion ZeChMEISTER et MUCINA 1994 suball, nov. (: 387, 400)

- Cardamino-Montenion ZECHMEISTER et MUCINA 1994 suball. nov. (: 400)

- Caricenion remotae ZECHMEISTER et MUCINA 1994 suball. nov. (: 401)

- Cratoneuro filicini-Calthenion laetae (HADAČ 1983) ZECHMEISTER et MUCINA 1994 suball. nov, et stat. nov. (: 401) 
- Lycopodio-Cratoneurenion commutati (HADAČ 1983) ZECHMEISTER et MUCINA 1994 suball. nov, et stat, nov. (: 400)

- Mniobryo-Epilobienion hornemannii (NORDHAGEN 1943) ZECHMEISTER in ZECHMEISTER et MUCINA 1994 suball. nov. et stat. nov. (: 400)

- Swertio-Anisothecienion squarrosi (HADAČ 1983) ZECHMEISTER in ZECHMEISTER et MUCINA 1994 suball. nov. et stat. nov. (: 400)

- Glycerietum nemoralis SANDA, POPESCU et SERBANESCU 1994 ass. nov. (: 14) [RO]

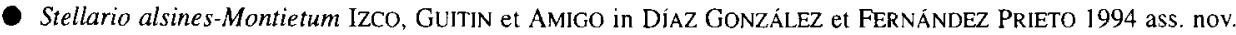
(: 499) [ES]

Mulgedio-Aconitetea

- Ligustico mutellinae-Luzulion desvauxii MiChalet et PHILIPPE 1994 all. nov. (: 418) [FR, "Massif Central"]

- Aconito neapolitani-Myrrhetum odorati Fernández Prieto et Nava in Díaz González et Fernández PRIETo 1994 ass. nov. (: 355) [ES]

O Heracleo sphondylii-Calamagrostietum arundinaceae MICHALET et PHILIPPE 1994 ass. nov., nom. inval. (: 414) [art. 5] [FR, "Massif Central"]

O Luzulo sylvaticae-Calamagrostietum arundinaceae MICHALET et PHILIPPE 1994 ass. nov., nom. inval. (: 414) [art. 5] [FR, "Massif Central"]

O Pediculari foliosae-Geranietum sylvatici Michalet et PhilipPe 1994 ass. nov., nom. inval. (: 421) [art. 5] [FR, "Massif Central"]

- Veratro albi-Luzuletum desvauxii (LuQuet 1926) MICHALET et PHILIPPE 1994 ass. nov (: 418, 421) [FR, "Massif Central"]

[sub: Veratro albi-Luzuletum desvauxii LUQUET $26 \mathrm{~cm}$. nov.]

Myrteolo-Sphagnetea

O Sphagno-Dacrydietum fonckii PISANO 1994 ass. nov., nom. prov. (: 11) [art. 3b] [CL, Chile South]

Nardeiea strictae

* Trifolio alpini-Meetalia athamantici DE FouCAULT 1994e ord. nov., nom. superfl. (: 437) [art. 29]

- Campanulo barbatae-Potentillion DE FouCAuLT I994e all. nov. (: 438)

O Campanulo herminii-Festucion eskiae DE FoUCAULT 1994e all. nov., nom. inval. (: 440) [art. 8] [ES]

- Carici arenariae-Festucion filiformis DE FOUCAULT 1994e all. nov. (: 435, 436)

O Carici macrostyli-Nardion strictae (RIVAS-MARTÍNEZ et al. 1984) DE FouCAULT 1994e all. nov., nom. inval. (: 438) [art. 8]

- Danthonio decumbentis-Serapion linguae DE FouCAULT 1994e all. nov. (: 447, 448)

O Galio idubidae-Nardion strictae (RIVAS-MARTÍNEZ) DE FOUCAULT 1994e all. nov., nom. prov. (: 435) [art. 3b] [ES]

$\triangle$ Galio saxatilis-Festucion filiformis (STIEPERAERE 1990) DE FOUCAULT 1994e all. nov. (: 434)

O Galio saxatilis-Festucion viviparae DE FoUCAULT 1994e all. nov., nom. inval. (: 436) [art. 8]

O Galio saxatilis-Potentillion aureae DE FOUCAULT 1994e all. nov., nom. inval. (: 437) [art. 8] [DE]

O Potentillion calabrae DE Foucault 1994e all. nov., nom. inval. (: 441) [art. 2b] [IT] [sub: Potentillion calabrae (BoNIN 1978) all. nov.]

- Thymelaeo dendrobryi-Nardetum DíAZ GonZÁlez et FERNÁNDEZ PRIETO 1994 ass. nov. $(: 351,352)$ [ES]

Ononido-Rosmarinete a (see also Rosmarinetea)

- Lavandulo mairei-Carthamion fruticosi QuÉzEL, BARBERO, BENABID et RIVAS-MARTíneZ I994 all. nov. (: 549 , 551) $[\mathrm{MA}]$

- Adenocarpo leiocarpi-Retametum dasycarpae QuÉzEL, BARBERO, BENABID et RIVAS-MARTÍNEZ I994 ass. nov. (: 558, 559) [MA]

- Convolvulo trabutiani-Genistetum capitellatae QUÉzEL, BARBERO, BENABID et RIVAS-MARTíNEZ 1994 ass. nov. (: 560, 561) [MA]

- Diantho turolensis-Genistetum hispanicae ROSELló GIMENO 1994 ass. nov. (: 418) [ES]

- Festuco hystricis-Genistetum eliassennenii Garcia Muangos, LOIDI et HerRera Gallastegui 1994 ass, nov. (: 101) [ES]

- Genisto myrianthis-Carthametum fruticosi QUÉZEL, BARBERO, BENABID et RIVAS-MARTÍNEZ 1994 ass. nov. (: 553 , 554) $[\mathrm{MA}]$ 
- Helianthemo mollis-Hypericetum ericoidis RosELLó GIMENO 1994 ass. nov. (: 421) [ES]

- Koelerio vallesianae-Thymetum mastigophori Garcia Muangos, LoIdi et Herrera Gallastegui 1994 ass. nov. (: 105) [ES]

- Lavandulo brevidentis-Hertietum maroccanae QuÉzEL, BARBERO, BENABID et RIVAS-MARTíNEZ 1994 ass. nov. (: 550, 551) [MA]

- Teucrio malenconiani-Thymetum brevidentis QUÉzEL, BARBERO, BENABID et RIVAS-MARTínEZ 1994 ass. nov. (: 563, 564) [MA]

- Thymo commutati-Rosmarinetum officinalis QUÉZEL, BARBERO, BENABID et RIVAS-MARTÍNEZ 1994 ass. nov. $(: 552,553)[\mathrm{MA}]$

- Ulici europaei-Genistetum occidentalis DÍAZ GONZÁLEZ et FERNÁNDEZ PRIETO 1994 ass. nov. (: 341, 345) [ES]

Onopordetea acanthii

- Carduo pycnocephali-Nicotianetum glaucae Biondi, BlASI, Brugiapaglia, Fogu et Mossa 1994 ass. nov. (: 307 ) [IT, Sardegna]

- Sylibo-Onopordetum arenarii GÉHU, KAABECHE et GHARZOULI 1994a ass. nov. (: 378, 380) [DZ]

- Verbasco montani-Onopordetum acanthii ROSELLÓ GIMENO 1994 ass. nov. (: 400) [ES]

Pegano-Salsoletea vermiculatae

$\triangle$ Artemision arborescentis GÉHU et BIONDI (1986) 1994 (: 123, 125) [FR, Corse]

- Artemision glutinosae-Hyssopetum cinerei RosElló GIMENo 1994 ass. nov. (: 403) [ES]

- Artemisio glutinosae-Gypsophiletum hispanicae ROSELló GIMENO 1994 ass. nov. (: 402) [ES]

- Artemisio herbae-albae-Santolinetum squarrosae LADERo ÁlvAREZ. VALLE GUTIÉREZ et GuTIÉREZ BALBAS 1994 ass. nov. (: 504, 505) [ES]

Pergulario tomentosae-Pulicarietea crispae

O Association à Ziziphus lotus et Coronilla juncea subsp. pomelii KAABECHE et GHARZOUL1 1994 ass. nov., nom. inval. (: 599) [art. 3h] [DZ]

- Rhantherio adpressi-Fagonietum zilloidis QUÉZEL, BARBERO, BENABID et RIVAS-MARTínEZ 1994 ass. nov. (: 573 , 574) $[\mathrm{MA}]$

Phlomidi lychnitidis-Brachypodietea retusi

- Phlomidi lychnitidis-Brachypodietea retusi Roselló GIMENO 1994 cl. nov. (: 411$)$

- Phlomidi lychnitidis-Brachypodietalia retusi ROSELLó GIMENO 1994 ord. nov. (: 413)

O Scabioso turolensis-Brachypodion retusi ROSELLó GIMENo 1994 all. nov., nom. inval. (: 413) [art. 5] [ES]

"Phellino-Rapaneetea macrophyllae"

O Phellino-Rapaneetea macrophyllae HoFf $1994 \mathrm{cl}$. nov., nom. prov. (: 682) [art. 3b] [NC]

O Archidendropsio-Agathidetalia lanceolatae HoF 1994 ord. nov., nom. prov. (: 682) [art. 3b] [NC]

O Austrobuxo-Nothofagetalia discoidis HoFF 1994 ord. nov., nom. prov. (: 682) [art. 3b] [NC]

O Archidendropsio-Agathidion lanceolatae HoFf 1994 all. nov., nom. prov. (: 682) [art. 3b] [NC]

O Austrobuxo-Nothofagion discoidis HoFF 1994 all. nov., nom. prov. (: 682) [art. 3b] [NC]

O Archidendropsio-Agathidetum lanceolatae HoFf 1994 ass. nov., nom. prov. (: 682) [art. 3b] [NC]

O Austrobuxo-Nothofagetum discoidis HoFf 1994 ass. nov., nom. prov. (: 682) [art. 3b] [NC]

Phragmito-Magnocaricetea

- Alismato michaletti-Sparganietum neglecti GÉHU, KAABECHE et GHARZOULI 1994c ass. nov. (: 303) [DZ]

O Caricetum kurdico-distichae OCAKVERDI 1994 ass. nov., nom. inval. (: 258, 261) [art. 5] [TR]

- Cladio marisci-Thelypteridetum interruptae GÉHU, KAABECHE et GHARZOULI 1994c ass. nov. (: 304) [DZ]

- Eleocharito-Sparganietum neglecti BRULLO, MINISSALE et SPAMPINATO 1994 ass. nov. (: 20) [IT]

- Helosciadio nodiflori-Alismetum michaletti GÉHU, KAABECHE et GHARZOULI 1994c ass. nov. (: 302) [DZ]

- Irido pseudacori-Caricetum lusitanicae BELlot ex DíaZ GONZÁLEZ et FernÁNDEZ PRIETo 1994 ass. nov. (: 492) [ES] 
O Lysimachio thyrsiflorae-Glycerietum declinatae JULVE et GILLET 1994 ass. nov., nom. prov. (: 56) [art. 3b] [PL]

- Panicetum repentis GÉHU, KAABECHE et GHARZOULı 1994c ass. nov. (: 304) [DZ]

O Ranunculo linguae-Caricetum acutiformis JULVE et GILLET 1994 ass. nov., nom. prov. (: 56) [art. 3b] [PL]

O Thelypterido palustris-Caricetum elongatae JULVE et GILLET 1994 ass. nov, nom. prov. (: 56) [art. 3b] [PL]

- Typhetum dominguensis Brullo, Minissale et SPAMPINATo 1994 ass. nov. (: 21) [IT]

Pino-Juniperetea

Festuco gautieri-Pinetum salzmannii RoSELLó GIMENO 1994 ass. nov. (: 434) [ES]

"Plantagini-Poetea flabellatae"

O Plantagini-Poetea flabellatae PISANO $1994 \mathrm{cl}$. nov., nom. prov. (: 13) [art. 3b] [CL, Chile South]

O Plantagini-Colobanthetalia quitensis PISANo 1994 ord. nov., nom. prov. (: 13) [art. 3b] [CL, Chile South]

O Poetalia flabellatae PISANO 1994 ord. nov., nom. prov. (: 13) [art. 3b] [CL, Chile South]

O Plantagini-Colobanthion quitensis PISANO 1994 all. nov., nom. prov. (: 13) [art. 3b] [CL, Chile South]

O Poion flabellatae PISANo 1994 all. nov., nom. prov. (: 13) [art. 3b] [CL, Chile South]

O Plantagini-Colobanthetum quitensis PISANO 1994 ass. nov., nom. prov. (: 13, 20) [art. 3b] [CL, Chile South]

O Poetum flabellatae PISANo 1994 ass. nov., nom. prov. (: 13, 20) [art. 3b] [CL, Chile South]

Potametea

- Alopecuro-Glycerion Brullo, Minissale et Spampinato 1994 all. nov. (: 14, 41) [IT]

O Potamion pusilli WIEgleb ex VAhLE in Preising, VAhle, Brandes, HofMeister, J. TüXen et Weber 1994 all nov., nom. inval. (: 110) [art. 2b] [DE]

O Utriculario minoris-Nymphaeion VAHLE in PREISING, VAHLE, BRANDES, HOFMEISTER, J. TÜXEN et WEBER 1994 all. nov., nom. inval. (: 110) [art. 2b] (: 113) [DE]

* Callitricho-Elodeetum canadensis PASSARGE (1964) 1994a nom. nov. (: 341) [art. 29]

O Ceratophyllo-Potametum lucentis (HUECK 1931) PASSARGE 1994a nom. nov., nom. inval. (: 343) [art. 2b]

O Charo-Potametum colorati (ALLORGE 1922) PASSARGE 1994a nom. nov., nom. inval. (: 357) [art. 2b]

O Elodeetum nuttallii (DE LANGE 1972) PASSARGE 1994a ass. coll. nov., nom. illeg. (: 342) [art. 3d, 39]

O Elodeo-Potametum alpini (KRAUSCH 1964) PODBIELKOWSKi (1967) PASSARGE 1994a nom. nov., nom. inval. (: 352, 353) [art. 2b]

O Elodeo-Potametum crispi (PIGNATti 1954) PASSARge 1994a ass. nov., nom. inval. (: 356) [art. 2b]

O Elodeo-Potametum praelongi (SAUER 1937) HLLd (1959) PASSARGE 1994a nom. nov., nom. inval. (: 350) [art. 2b]

- Glycerio-Callitrichetum obtusangulae BRULLO, MinisSALE et SPAMPINATo 1994 ass. nov. (: 17) [IT]

- Glycerio spicatae-Oenanthetum aquaticae BRULLO, MINISSALE et SPAMPINATO 1994 ass. nov. (: 16, 17) [IT]

O Groenlandio-Potametum nitentis (KOCH 1926) PASSARGE 1994a nom. nov., nom. inval. (: 362) [art. 2b]

O Isolepido-Potametum graminei (MANEGOLD 1977) PASSARGE 1994a nom. nov., nom. inval. (: 359, 360) [art. 2b]

- Myriophyllo spicati-Potametum nodosi GÉHU, KAABECHE et GHARZOUL1 1994c ass. nov. (: 303) [DZ]

O Myriophyllo verticillati-Hydrilletum (TOMASZIEWICZ 1979) PASSARGE 1994a nom. nov., nom. inval. (: 340) [art. 2b]

O Nupharo-Potametum lucentis PASSARGE (1964) 1994a ass. nov., nom. inval. (: 346) [art. 2b]

O Nupharo-Potametum perfoliati (ZONNEVELD 1960) ARENDT (1982) PASSARGE 1994a nom. nov., nom. inval. (: 349) [art. 2b]

O Polygono-Potametum graminei PASSARGe (1964) 1994a ass. nov., nom. inval. (: 360,361 ) [art. 2b]

O Potametum crispo-lucentis PASSARGE 1994a ass. nov., nom. inval. (: 345) [art. 2b] [DE]

- Potametum filiformi-graminei PASSARge 1994a ass. nov. (: 361) [DE] [sub: Potametum filiformi-graminei PASSARGE (1963) comb. nov.]

O Potametum filiformi-nitentis PASSARGE (1963) 1994a nom. nov., nom. inval. (: 364) [art. 2b]

O Potametum friesii-nitentis (MICHNA 1976) PASSARge 1994a nom. nov., nom. inval. (: 364) [art. 2b]

O Potametum natanti-crispi (KAISER 1926) PASSARGE 1994a nom. nov., nom. inval. (: 356) [art. 2b]

O Potametum pectinato-nodosi (KNAPP et STOFFERS 1962) PASSARGE 1994a ass. nov., nom. inval. (: 353, 354) [art. 2b]

O Potametum perfoliato-alpini (MICHNA 1976) PASSARGE 1994a ass. nov., nom. inval. (: 351) [art. 2b]

O Potametum perfoliato-nitentis (MICHNA 1976) PASSARge 1994a nom. nov., nom. inval. (: 364) [art. 2b]

O Potamogeton filiformis-Potamogeton perfoliatus-Association PASSARGE 1994a ass. nov., nom. inval. (: 349) [art. 2b, 3h] [CH, DE]

O Ranunculo-Elodeetum canadensis (VAN DONSElAAR 1961) PASSARGE 1994a ass. nov., nom. inval. (: 342) [art. 2b]

O Ranunculo trichophylli-Groenlandietum (KOHLER et al. 1974) PASSARGE 1994a ass. nov., nom. inval. (: 354) [art. $2 b]$ 
O Utriculario-Potametum polygonifolii (CHOUARD 1926) PASSARge 1994a ass. nov., nom. inval. (: 366) [art. 2b]

- Wolffio-Utricularietum neglectae GÉHU, KAABECHE et GHARZOULi 1994c ass. nov. (: 303) [DZ]

- Zanichellio-Myriophylletum alterniflori GÉHU, KAABECHE et GHARZOULI 1994c ass. nov. (: 301) [DZ]

"Protosparago africani-Acacion karroo"

O Protosparago africani-Acacion karroo BEZUIDENHOUT, BREDENKAMP et THERON $1994 \mathrm{c}$ all nov., nom. inval. (: 216) [art. 5] [ZA, Transvaal]

- Eragrostio curvulae-Acacietum karroo BEzUIDENhOUT, BREDENKAMP et THERON 1994c ass. nov. (: 216) [ZA, Transvaal]

- Protosparago suaveolentis-Acacietum karroo BezUidenhout, BredenKamp et Theron 1994c ass. nov. (: 216) [ZA, Transvaal]

- Rhoo lanceae-Acacietum caffrae BEZUidenhout, BREDENKAMP et THERON 1994c ass. nov. (: 221) [ZA, Transvaal]

Pterido-Eriaxietea rigidae

O Baeckeo-Tieghemopanacetalia harmsii HoFf 1994 ord. nov., nom. prov. (: 681) [art. 3b] [NC]

O Costulario-Schoenetalia brevifolii HoFF 1994 ord. nov., nom. prov. (: 682) [art. 3b] [NC]

O Baeckeo-Tieghemopanacion harmsii HoFf 1994 all. nov., nom. prov. (: 681) [art. 3b] [NC]

O Codio-Hibbertion pancheri HoFF 1994 all. nov., nom. prov. (: 682) [art. 3b] [NC]

O Costulario-Schoenion brevifolii HOFF 1994 all. nov., nom. prov. (: 682) [art. 3b] [NC]

O Costulario-Styphelion albicantis (JAFFRÉ 1980) HOFF 1994 all. nov., nom. prov. (: 681) [art. 3b] [NC]

O Knightio-Argophillion laxi (JAFFRÉ 1980) HOFF 1994 all. nov., nom. prov. (: 681) [art. 3b] [NC]

O Alixio-Styphelietum cymbulae HoFf 1994 ass. nov., nom. prov. (: 682) [art. 3b] [NC]

O Baeckeo-Costularietum comosae Hoff 1994 ass. nov., nom. prov. (: 681) [art. 3b] [NC]

O Balanopsio-Hibbertietum brongniartii HoFf 1994 ass. nov., nom. prov. (: 682) [art. 3b] [NC]

O Costulario-Styphelietum albicantis (JAFFRÉ 1980) HOFF 1994 ass, nov., nom. prov. (: 681) [art. 3b] [NC]

O Dendrobio-Agathidetum ovatae HoFf 1994 ass. nov., nom. prov. (: 682) [art. 3b] [NC]

O Hibbertio-Garcinietum amplexicaulis HoFf 1994 ass. nov., nom. prov. (: 681) [art. 3b] [NC]

O Knightio-Argophilletum laxi (JAFFrÉ 1980) HOFF 1994 ass. nov., nom. prov. (: 681) [art. 3b] [NC]

O Normandio-Hibbertietum altigenae HoF 1994 ass. nov., nom. prov. (: 681) [art. 3b] [NC]

O Pancherio-Baeckeetum ericoidis HoFf 1994 ass. nov, nom. prov. (: 682) [art. 3b] [NC]

O Pancherio-Casuarinetum deplanchei HoFf 1994 ass. nov., nom. prov. (: 682) [art. 3b] [NC]

O Rapaneo-Araucarietum laubenfelsii HoFF 1994 ass. nov., nom. prov. (: 681) [art. 3b] [NC]

O Rapaneo-Araucarietum rulei (JAFFRÉ 1980) HOFF 1994 ass. nov., nom. prov. (: 682) [art. 3b] [NC]

Quercetea ilicis

- Calluno vulgaris-Lauretum nobilis Fernández Prieto, Arbesú et Bueno in Díaz González et Fernández PRIETO 1994 ass. nov. (: 323) [ES]

O Clematido cirrhosae-Pistacietum lentisci GamiSANS et MURACCIOLE 1985 corr. superfl. in GÉHU et B1ONDI 1994 (: 111, 113) [art. 14, 40]

- Frangulo alni-Arbutetum unedonis DíAz GonZÁlez et FERnÁndez PRIETo 1994 ass. nov. (: 321,323 ) [ES]

- Genisto occidentalis-Quercetum cocciferae LOIDI, HERRERA, OLANO et SILVÁN 1994 ass. nov. (: 536-537) [ES]

- Junipero macrocarpae-Euphorbietum dendroidis GÉHU et BIONDI 1994 ass. nov. (: 113) [FR, Corse]

- Lauro-Quercetum pubescentis BARBERO et QUÉZEL 1994 ass. nov. (: 84) [FR]

- Lonicero implexae-Quercetum rotundifoliae LOUSÃ, ESPRITO SANTO et COSTA 1994 ass. nov. (: 222) [PT]

- Phillyreo latifoliae-Arbutetum unedonis LOIDI, HERRERA, OLANO et SILVÁN 1994 ass. nov. (: 536-537) [ES]

- Rhamno saxatilis-Juniperetum phoeniceae ROSEL LÓ GIMENO 1994 ass. nov. (: 445) [ES]

O Rubio longifoliae-Juniperetum oxycedri COSTA, CAPELO, LOUSÃ et AGUIAR 1994 ass. nov., nom. prov. [art. 3b] (: 509) $[\mathrm{PT}]$

- Taxo-Quercetum ilicis BARBERo et QuÉZEL 1994 ass. nov. (: 83) [FR]

- Tetraclinido articulatae-Quercetum cocciferae BENABID et FENNANE 1994 ass. nov. (: 42) [MA]

- Ulici gallii-Arbutetum unedonis LOIDI, HERRERA, OLANO et SILVÁN 1994 ass. nov. (: 536-537) [ES]

- Zizipho loti-Rhoetum tripartiti QUÉZEL, BARBERO, BENABID et RIVAS-MARTÍNEZ 1994 ass. nov. (: 543, 544) [MA]

Quercetea pubescentis (see also Querco-Fagetea)

O Arabido-Juniperetum excelsae SERIN et EYCE 1994 ass. nov., nom. inval. (: 220, 222) [art. 3g] [TR]

O Astragalo-Pinetum brutiae SERIN et EYCE 1994 ass. nov. (: 206, 212) [art. 3g] [TR] 
- Astragalo podperi-Juniperetum foetidissimae TATLI, EYCE et SERIN 1994 ass. nov. (: 270, 278) [TR]

- Cicerbito-Cedretum libani SERIN et EYCE 1994 ass. nov. (: 215, 219) [TR]

- Diantho micranthi-Pinetum nigrae TATLI, EYCE et SERIN 1994 ass. nov. (: 269, 270) [TR]

- Genisto-Styracetum officinalis SERIN et EYCE 1994 ass. nov. (: 205, 209) [TR]

- Iberido-Abietetum cilicicae SERIN et EYCE 1994 ass. nov. (: 215, 217) [TR]

- Ilici-Quercetum pubescentis BARBERo el QUÉzEL 1994 ass. nov. (: 85) [FR]

- Lathyro digitati-Quercetum cerridis TATLI, EYCE et SERIN 1994 ass. nov. (: 270) [TR]

- Quercetum boissierii BEHÇET 1994 b ass. nov. (: 292, 293) [TR]

O Rubo incanescentis-Quercetum canariensis QUÉZEL 1956 nom. mut., nom. illeg. in MEDDOUR 1994 (: 49) [art. 45]

O Salvio-Quercetum cocciferae SERIN et EXCE 1994 ass. nov., nom. inval. (: 205, 206) [art. 3g] [TR]

- Salvio tomentosae-Cistetum laurifolii TATLI, EYCE et SERIN 1994 ass. nov. (: 278, 281) [TR]

- Seslerio albicantis-Quercetum pubescentis CHYTRÝ 1994 ass. nov. (: 123, 131) [SK]

O Sileno-Pinetum nigrae SERIN et EYCE 1994 ass. nov., nom. inval. (: 206, 214) [art. 3g] [TR]

$Q$ uercetea robori-petraeae

O Carici arenariae-Betuletum RINGER 1994 ass. nov., nom. inval. (: 128) [art. 3g] [DE, "Borkum", “Norderney"]

Querco-Fagetea (see also Quercetea robori-petraeae)

- Buxo-Fagion BARBERo et QuÉżL 1994 all. nov. (: 87, 88) [FR]

O Ulmo glabrae-Tilienion cordatae KIELLAND-LUND 1994 suball. nov., nom. inval. (: 308) [art. 5] [NO]

O Allio montani-Quercetum pubescentis STEIGER 1994 ass. nov., nom. prov. (: 197) [art. 3b] [CH]

O Alno viridis-Sorbetum aucupariae STEIGER 1994 ass. nov, nom. prov. (: 96) [art. 3b] [CH]

- Amelanchiero-Pinetum mugo MiNGHeTtr in PEDROTtI 1994 ass. nov. (: 134, 137) [IT, "Alps"]

- Cotino-Amelanchieretum ovalis Pedrotti et Minghetti in PEdrotti 1994 ass. nov. (: 45) [IT, "Alps"]

O Cytiso sessilifolii-Amelanchieretum ovalis PEDROTTI, GAFTA et MINGHETTI in PEDROTTI 1994 ass. nov., nom. illeg. (: 11) [Art, 31] [IT, "Alps"]

$\triangle$ Dryopterido carthusianae-Fagetum (Burduja, MiHAi et SÂRbu 1972) CHIFU et ŞTEFAN 1994 nom. nov. (: 72) [RO]

- Festuco giganteae-Fraxinetum excelsioris FERnÁndez PRIETo et BUENo in Díaz GonzÁlez et FERnÁNDEZ PRIETo 1994 ass. nov. (: 299, 300) [ES]

- Galio odorati-Abietetum GAFTA 1994 ass. nov. (: 58, tab. 11) [IT, "Alps"]

$\Delta$ Galio schultesii-Fagetum (BURduja, MiHAI et SÂRBU 1972) CHIFU et \$TEFAN 1994 nom. nov. (: 72) [RO]

$\triangle$ Hacquetio epipactidis-Fraxinetum MARINCÉ ex POLDINI et NARDINI 1994 ass. nov. (: 225) [IT]

- Humulo lupuli-Alnetum glutinosae BIURRUN, GARCía-MiJANGos et Loidr 1994 ass. nov. (: 46) [ES]

- Luzulo henriquesii-Aceretum pseudoplatani FERNÁNdez PRIETo et BUENo in Díaz GonzÁlez et FERNÁNDEZ PRIETO 1994 ass. nov. (: 294) [ES]

- Luzulo henriquesii-Quercetum petraeae (FERnÁndez Prieto et VÁsquez 1987) Díaz GonzÁlez et FernáNDEZ PRIETO 1994 ass. nov. (: 293) [ES]

- Luzulo niveae-Abietetum GAFTA 1994 ass. nov. (: 58, tab. 14) [IT, "Alps"]

$\triangle$ Polysticho lonchitis-Fagetum (HORVAT 1938) MARINČEK in POLDINI et NARDINI 1994 ass. nov. (: 248) [IT]

O Rubio peregrinae-Populetum albae VARESE 1994 ass. nov., nom. prov. (: 582, 584, 585) [art. 3b] [FR]

- Salicetum angustifolio-albae DíAZ GONZÁlez et FERnÁNDEZ PRIETo 1994 ass. nov. (: 302, 303) [ES]

O Salici-Populetum VARESE 1994 ass. nov., nom. prov. (: 582, 584, 585) [art. 3b] [FR]

- Smilaco-Arbutetum unedonis DÍAZ GonzÁleZ et FernáNDEZ PRIETo 1994 ass. nov. (: 308,310$)$ [ES]

Rhamno-Prunet e a (see also Querco-Fagetea)

O Carpino-Prunenion spinosae Delelis-Dusollier, Botineau, WaTtez-Franger et GheSTEM 1994 suball. nov., nom. inval. (: 516) [art. 8] [FR]

- Erico scopariae-Spiraeetum obovatae BotINEAU et GHESTEM 1994 ass. nov. (: 335) [FR]

O Evonymo-Ribetum alpini Delelis-Dusolller, BotineAU, WATTEZ-FranGER et GHESTEM 1994 ass. nov.[?], nom. inval. (: 516) [art. 5] [FR]

- Rhamno saxatilis-Ericetum scopariae BoTINEAU et GHESTEM 1994 ass. nov. (: 335) [FR]

"Rhoo leptodictyo-Acacion caffrae"

O Rhoo leptodictyo-Acacion caffrae BEZUIDENHOUT, BREDENKAMP et THERON 1994a all. nov., nom. inval. (: 74) [art. 5, 17] [ZA] 
Acacietum karroo-caffrae BEZUIDENHOUT, BREDENKAMP et THERON 1994a ass. nov. (: 74) [ZA]

O Dombeyo rotundifoliae-Acacietum caffrae BEZUIDENHOUT, BREDENKAMP et THERON 1994a ass. nov. (: 79) [art. 5] [ZA]

- Proteo caffrae-Acacietum caffrae BEZUIDENHOUT, BREDENKAMP et THERON 1994a ass. nov. (: 79) [ZA]

- Rhoo rigidae-Acacietum caffrae BEZUIDENHOUT, BREDENKAMP et THERON 1994a ass. nov. (: 79) [ZA]

Rosmarinetea

- Asperulo hirsutae-Ulicetum scabri RIVAS-MARTÍNEZ in DiÉz GARRETAS et ASENSI MARFIL 1994 ass. nov. (: 543 ) [ES]

- Astragalo massiliensis-Genistetum corsicae GÉHU et BIONDI 1994 ass. nov. (: 106) [FR, Corse]

- Sideritido chamaedryfoliae-Teucrietum dunensis DE LA TORRE et ALCARAZ 1994 ass. nov. (: 131) [ES]

- Teucrio lusitanici-Coridothymetum capitati (RIVAS GODAY et RIVAS-MARTínEZ 1969) RIVAS-MARTÍNEZ in DIÉZ Garretas et AsENSi Marfil 1994 nom. nov. (: 542)

- Ulici baetici-Cistetum clusii Rivas Goday et Rivas-Martínez 1969 cort. ASENSI MARFL et Diéz GarReTAS in Diéz GaRRETas et ASENSI MARFIL 1993 (: 542)

Ruderali-Manihotetea

- Sido-Sennion obtusifoliae SINSIN 1994 all. nov. (: 94) [BJ]

O Setario-Sporoboletum pyramidalis SINSIN 1994 ass. nov., nom. inval. (: 94, 99) [art. 3g] [BJ]

Ruderali-Secalietea

- Artemision variabilis BIONd, Ballelli, Allegrezza, TAFFETaNi et FranCalancia 1994 all. nov. (: 55) [IT]

O Stipion capensis BR.-BL. ex Bolòs 1956 nom. mut., nom. illeg. in CANO CARMONA et GaRCÍA FuENTES 1994 (: 60) [art. 45]

- Arenario hispanicae-Chamaemeletum fuscati GALÁN DE MERA 1994 ass. nov. (: 114) [ES]

O Biscutello auriculatae-Ericetum longirostris RIVAS-MARTÍNEZ 1978 nom. mut., nom. illeg. in CANO CARMONA et GARCÍA FUENTES 1994 (: 50) [art. 45]

- Convolvulo cupaniani-Malopetum trifidae GALÁN DE MERA 1994 ass. nov. (: 120) [MA]

- Conyzo canadensis-Chenopodietum botryos BIONDI, BALlelli, Allegrezza, TAFFETANi et FAnCALANCIA 1994 ass. nov. (: 53) [IT]

O Myosotido discolori-Brassicetum barrelieri RIVAS GODAY 1964 nom. mut., nom. illeg. in CANO CARMONA et GARCia Fuentes 1994 (: 65) [art. 45]

- Trifolietum angustifolio-glomerati ROSELLó GIMENO 1994 ass. nov. (: 397) [ES]

Saginetea maritimae

- Desmazerio marini-Brometum ferronii BIORET 1994 ass. nov. (: 134) [FR]

- Limonio avei-Hymenolobetum procumbentis BRULLO, SCELSI et SIRACUSA 1994 [1995?] ass. nov. (: 354) [IT, Sicilia]

- Limonio-Parapholidetum marginatae BRULLO, SCELSI et SiRACUSA 1994 [1995?] ass. nov. (: 354) [IT, Sicilia]

- Parapholidetum filiformis BRULlo, SCELSI et SIRACUSA 1994 [1995?] ass. nov. (: 358) [IT, Sicilia]

Salicetea purpureae

O Alno incanae-Salicetum albae VARESE 1994 ass. nov., nom. inval. (: 576, 577) [art. 5] [FR]

O Brachypodio phoenicoidis-Populetum nigrae VARESE 1994 ass. nov., nom. inval. (: 580, 581, 583) [art. 5] [FR]

O Corylo-Populetum nigrae VARESE 1994 ass. nov, nom. inval. (: 575, 581) [art. 5] [FR]

O Phalarido-Populetum nigrae VARESE 1994 ass. nov., nom. inval. (: 580, 583) [art. 5] [FR]

Salicornietea

$\triangle$ Agropyretalia pycnanthi GÉHU 1968 corr. DÍAZ GONZÁLEZ et FERnÁndez PRIETO 1994 (: 469$)$

$\triangle$ Agropyrion pycnanthi GéHu 1968 corr. DÍAz González et Fernández PriETo 1994 (: 469 )

O Puccinellio maritimae-Halimionion portulacoidis GÉHU 1994 all. nov., nom. inval. (: 194) [art. 5, 8]

- Puccinellietum giganteae BEHÇET 1994a ass. nov. (: 237, 238) [TR] 
"Scaevolo-Casuarinetea equisetifoliae"

O Scaevolo-Casuarinetea equisetifoliae HoFf 1994 cl. nov., nom. prov. (: 678) [art. 3b] [NC]

O Scaevolo-Casuarinetalia equisetifoliae HoFf 1994 ord. nov., nom. prov. (: 678) [art. 3b] [NC]

O Scaevolo-Casuarinion equisetifoliae HOFF 1994 all. nov., nom. prov. (: 678) [art. 3b] [NC]

O Loto-Wedelietum uniflorae HoFf 1994 ass. nov., nom. prov. (: 678) [art. 3b] [NC]

O Wedelio-Casuarinetum equisetifoliae HoFF 1994 ass. nov., nom. prov. (: 678) [art. 3b] [NC]

Scaevolo-Ipomoeetea pedis-caprae

O Scaevolo-Ipomoeetalia OHBA 1994 ord. nov., nom. prov. (: 50) [art. 3b] [MP]

O Ipomoeion pedis-caprae OHBA 1994 all. nov., nom. prov. (: 50) [art. 3b] [MP]

O Capparidetum cordifoliae OHBA 1994 ass. nov., nom. inval. (: 32) [art. 5] [MP]

O Crinetum asiaticae OHBA 1994 ass. nov., nom. inval. (: 36) [art. 5] [MP]

O Fimbristylidetum cymosae OHBA 1994 ass. nov., nom. inval. (: 46) [art. 5] [MP]

O Portulacetum luteae OHBA 1994 ass. nov., nom. inval. (: 44) [art. 5] [MP]

O Zoysietum matrellae OHBA 1994 ass. nov., nom. inval. (: 35) [art. 5] [MP]

Scheuchzerio-Caricetea fuscae

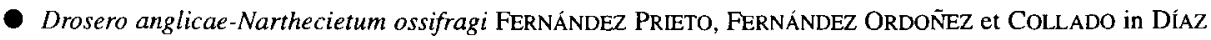
GONZÁlez et FERnÁNDEZ PRIETo 1994 ass. nov. (: 483) [ES]

Sedo-Sclerant he te a (see also Koelerio-Corynephoretea)

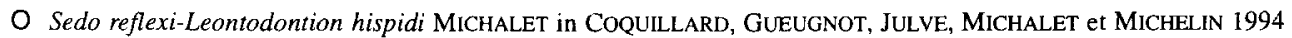
all. nov., nom. prov. (: 43) [art. 3b] [FR]

O Euphorbio truncatae-Cladonietum symphicarpae BRAQUE et LOISEAU 1994 ass. nov., nom. inval. (: 100) [art. 5] [FR]

O Peltigero rufescentis-Allietum sphaerocephali BRAQUE et LOISEAU 1994 ass. nov., nom. inval. (: 34) [art. 5] [FR]

- Sedo anglici-Scilletum vernae BIORET 1994 ass. nov. (: 131) [FR]

O Vulpio unilateralis-Desmazerietum rigidi BRAQUE et LOISEAU 1994 ass. nov., nom. inval. (: 98) [art. 5] [FR]

Seslerietea albicantis (see Elyno-Seslerietea)

Sido-Stachytarphetetea indicae

O Nephrolepido-Polygaletalia paniculatae Hoff 1994 ord. nov., nom. prov. (: 683) [art. 3b] [NC]

O Stachytarpheto-Melastometalia malabathrici HoFf 1994 ord. nov., nom. prov. (: 684) [art. 3b] [NC]

O Dicranopterido-Melastomion malabathrici HoF 1994 all. nov., nom. prov. (: 684) [art. 3b] [NC]

O Nephrolepido-Polygalion paniculatae HoFf 1994 all. nov., nom. prov. (: 683) [art. 3b] [NC]

O Paspalo-Elephantopion mollis HoFf 1994 all. nov., nom. prov. (: 684) [art. 3b] [NC]

O Cyatheo-Rubetum roseafolii HoFf 1994 ass. nov., nom. prov. (: 684) [art. 3b] [NC]

O Dicranopterido-Melastometum malabathrici Hoff 1994 ass. nov., nom. prov. (: 684) [art. 3b] [NC]

O Guioo-Nephrolepidetum cordifoliae HoFf 1994 ass. nov., nom. prov. (: 683) [art. 3b] [NC]

O Lantano-Polygaletum paniculatae HoFf 1994 ass. nov., nom. prov. (: 683) [art. 3b] [NC]

O Paspalo-Elephantopetum mollis HoF 1994 ass. nov., nom. prov. (: 684) [art. 3b] [NC]

O Sido-Stenotaphretum dimidiati HOFF 1994 ass. nov., nom. prov. (: 684) [art. 3b] [NC]

Soncho-Bidentetea

O Spermacocetalia stachydeae SINSIN 1994 ord. nov., nom. inval. (: 95) [art. 5] [BJ]

- Andropogonion pseudocaprici SINSIN 1994 all. nov. (: 96) [BJ]

- Kohaution grandiflorae SINSIN 1994 all. nov. (: 95) [BJ]

O Spermacoco-Pennisetion polystachii SINSIN 1994 all. nov., nom. inval. (: 95) [art. 8] [BJ]

- Celosietum trigynae SINSIN 1994 ass. nov. (: 95, 100) [BJ]

- Desmodietum hirti SINSIN 1994 ass. nov. $(: 96,101)$ [BJ] 
O Rivino-Passifloretum suberosae HoFF 1994 ass. nov., nom. prov. (: 684) [art. 3b] [NC]

O Urochloo-Tephrosietum pedicellatae SINSIN 1994 ass. nov., nom. inval. (: 96, 100) [art. 3g] [BJ]

Spartocytisetea supranubit

- Spartocytisetea supranubii SCHÖNFELDER et VOGGENREITER $1994 \mathrm{cl}$. nov. (: 481 ) [ES, Canary Is]

- Spartocytisetalia supranubii SCHÖNFELDER et VoGgENREITER 1994 ord. nov. (: 481) [ES, Canary Is]

- Echietum auberiani SCHÖNFELDER et VOGGENREITER 1994 ass. nov. (: 470) [ES, Canary Is]

"Stenotaphro-Acacietea simplicis"

O Stenotaphro-Acacietea simplicis HoFf $1994 \mathrm{cl}$ nov., nom. prov. (: 679) [art. 3b] [NC]

O Leucaeno-Vitietalia trifoliatae HoFf 1994 ord. nov., nom. prov. (: 679) [art. 3b] [NC]

O Stenotaphro-Scaevoletalia sericeae HoFf 1994 ord. nov., nom. prov. (: 679) [art. 3b] [NC]

O Cenchro-Ipomoeion pedis-caprae HoFf 1994 all. nov., nom. prov. (: 679) [art. 3b] [NC]

O Lepturo-Scaevolion sericeae HoFf 1994 all. nov., nom. prov. (: 679) [art. 3b] [NC]

O Leucaeno-Chlorision virgatae HoFF 1994 all. nov., nom. prov. (: 679) [art. 3b] [NC]

O Stenotaphro-Acacion simplicis HoFF 1994 all. nov., nom. prov. (: 679) [art. 3b] [NC]

O Stenotaphro-Derrision trifoliolatae HoFf 1994 all. nov., nom. prov. (: 679) [art. 3b] [NC]

O Lepturo-Cenchretum echinatae HoFF 1994 ass. nov., nom. prov. (: 679) [art. 3b] [NC]

O Lepturo-Ipomoeetum pedis-caprae HoFf 1994 ass. nov., nom. prov. (: 679) [art. 3b] [NC]

O Lepturo-Scaevoletum sericeae HoFF 1994 ass. nov., nom. prov. (: 679) [art. 3b] [NC]

O Leucaeno-Chlorisetum virgatae Hoff 1994 ass. nov., nom. prov. (: 679) [art. 3b] [NC]

O Sporobolo-Acacietum simplicis HOFF 1994 ass. nov., nom. prov. (: 679) [art. 3b] [NC]

O Stenotaphro-Abutiletum indicae HoFf 1994 ass. nov., nom. prov. (: 679) [art. 3b] [NC]

O Stenotaphro-Derrisetum trifoliolatae Hoff 1994 ass. nov., nom. prov. (: 679) [art. 3b] [NC]

Stellarietea mediae (see also Chenopodietea)

- Bromo rubentis-Lamarckietum aureae BIONDI, BLASI, BRUGIAPAGLIA, FOGU et MOSSA 1994 ass. nov. (: 306) [IT, Sardegna]

- Lactuco serriolae-Atriplicetum sagittatae (KNAPP ex SCHREIER 1955) WIBKIRCHEN et KRAUSE 1994 nom. nov. (: 434)

- Sileno gallicae-Brometum gussonei GÉHU et BIONDI 1994 ass. nov. (: 54) [FR, Corse]

- Vallantio-Solenopsidetum annuae BRULLo, SCELSI et SIRACUSA 1994 [1995?] ass. nov. (: 362) [IT, Sicilia]

Stipo-Trachynietea distachyae

Atractylido-Neatostemetum apuli BRullo, SCElSI et SiRACUSA 1994 [1995?] ass. nov. (: 352) [IT, Sicilia]

Thero-Salicornietea

O Halocnemion strobilacei (GÉHU et COSTA 1984) ABBAS et GÉHU 1994 all. nov. et stat. nov., nom. inval. (: 113) [art. 2b]

- Salicornio patulae-Crypsietum aculeatae PARADIS et LORENZONI 1994 ass. nov. (: 20) [FR, Corse]

- Scorzoneretum parviflorae BEHÇET 1994a ass. nov. (: 238, 239) [TR]

Thlaspietea rotundifoli

- Iberido-Linarion propinquae DÍAZ GONZÁlez et FERNÁNDEZ PRIETo 1994 all. nov. (: 445) [ES]

- Aquilegienion bertolonii TOMASELLI 1994 suball. nov. (: 41, 46) [IT]

- Arabidenion alpinae (BÉGUIN 1972) EGGENSBERGER 1994 suball. nov. et stat. nov. (: 77) [recte: BÉGUIN 1974]

- Petasitenion paradoxi EgGENSBERGER 1994 suball. nov. (: 84) [sub: Petasitenion paradoxi ZOLLITSCH 66]

- Athamanto cretensis-Trisetetum argentei POLDINI et MARTINI 1994 ass. nov. (: 172) [IT]

- Hieracietum intybacei POLDINI et MARTINI 1994 ass. nov. (: 153) [IT] 
- Linario glabrescentis-Rumicetum suffruticosi DIAZ GonZÁlez et FERNÁNDEZ PRIETo 1994 ass. nov. (: 450, 451) [ES]

O Saxifrago-Leontodontetum hyoseroidis TOMASELLt 1994 ass. nov., nom. inval. (: 41, 46) [art. 3g] [IT]

"Trachypogono spicati-Diheteropogonion amplectentis"

O Trachypogono spicati-Diheteropogonion amplectentis BEZUIDENHOUT, BREDENKAMP et THERON 1994b all. nov., nom. inval. (: I59) [Art. 5, 17] [ZA, Transvaal]

- Alloteropsido semialatae-Tristachyetum leucothricis BEZUIDENHOUT, BREDENKAMP et THERON 1994b ass. nov. (: 159) [ZA, Transvaal]

- Loudetio simplicis-Diheteropogonetum amplectentis BEZUIDENHOUT, BREDENKAMP et THERON 1994b ass. nov. (: 159) [ZA, Transvaal]

Trifolio-Geranietea

O Centaureo lugdunensis-Melampyretum cristati BRAQUE et LOISEAU 1994 ass. nov., nom. inval. (: 34) [art. 5] [FR]

O Euphorbio angulatae-Spiraeetum obovatae BRAQUE et LOISEAU 1994 ass. nov., nom. prov. (: 34) [art. 3b] [FR]

- Fragario-Trifolietum rubentis PASSARGE 1994b ass. nov. (: 170) [DE]

O Odontito chrysanthae-Phyteumatetum teneri BRAQUE et LOISEAU 1994 ass. nov., nom. inval. (: 154) [art. 5] [FR]

- Sanguisorbo-Silenetum nutantis PASSARGE 1994b ass. nov. (: 170) [DE]

- Veronico chamaedryos-Hieracietum laevigatae PASSARGE 1994c ass. nov. (: 93) [DE]

O Veronico-Peucedanetum cenvariae PASSARGE 1994b ass. nov., nom. inval. (: 168) [art. 3g] [DE]

Tuberarietea guttatae

- Laguro-Vulpion fasciculatae GÉHU et BIONDI 1994 all. nov. (: 51) [FR, Corse]

- Ammochloo palaestinae-Linarietum nigricantis MOTA, CABELlo, GómEZ MERCADO et PEÑAs 1994 ass. nov. (: 54) [ES]

- Bellidi-Solenopsidetum laurentiae BRULLo, SCELSI et SIRACUSA 1994 [1995?] ass. nov. (: 348) [IT, Sicilia]

- Bupleuro-Ononidetum reclinatae BRULLO, SCELSI et SIRACUSA 1994 [1995?] ass. nov. (: 350) [IT, Sicilia]

- Cerastio taurici-Myosotidetum ramosissimae ROSELLó GIMENo 1994 ass. nov. (: 405) [ES]

- Corrigiolo telephifoliae-Corynephoretum articulati GÉHU, BIONDI, GÉHU-FRANCK et TAFFETANI ex GÉHU et BIONDI 1994 ass. nov. (: 44) [FR, Corse]

- Cutandietum maritimae PIAzZA et PARAdis 1994 ass. nov. (: 74) [FR, Corse]

O Helianthemo angustati-Euphorbietum sulcatae GARCÍA Río et NAVARRO ANDRÉS 1994 ass. nov., nom. prov. (: I65) [art. 3b] [ES]

- Herniario-Crassuletum tillaeae Brullo, SCElsi et SIRACuSa 1994 [1995?] ass. nov. (: 349) [TT, Sicilia]

- Iflogo spicatae-Silenetum adscendentis MOTA, CABELlo, GómEZ MERCADO et PEÑAS 1994 ass. nov. (: 54, 57) [ES]

* Senecioni leucanthemifolii-Matthioletum tricuspidatae (PARADIS et PIAZZA 1992) GÉHU et BIONDI 1994 ass. nov., nom. superfl. (: 43, 44) [art. (25) 29] [FR, Corse]

- Sileno gallicae-Corynephoretum articulati GÉHU et BIONDI 1994 ass. nov. (: 49) [FR, Corse]

- Sileno nicaeensis-Cutandietum maritimae GÉHU et BIONDI 1994 ass. nov. (. 41) [FR, Corse]

- Sileno nicaeensis-Ononidetum diffusae PARADIS et PIAZZA ex GÉHU et BIONDI 1994 ass. nov. (: 49) [FR, Corse]

O Sileno nicaeensis-Ononidetum variegatae (GÉHU, BIONDI, GÉHU-FrANCK et ARNOLD-APOSTOLIDES 1986) GÉHU et BIONDI 1994 ass. nov., nom. illeg. (: 38, 41) [art. 31] [FR, Corse]

- Sileno nicaeensis-Vulpietum fasciculatae GÉHU et BIONDI 1994 ass. nov. (: 51) [FR, Corse]

- Sileno sericeae-Cutandietum maritimae GÉHU et BIONDI 1994 ass. nov. (: 41) [FR, Corse]

O Sileno sericeae-Ononidetum variegatae PARADIS et PIAZZA ex GÉHU et BIONDI 1994 ass. nov., nom. inval. (: 41, 147) [art. (1), 6] [FR, Corse]

- Tuberario-Anemonetum palmatae BRULlo, SCELSI et SIRACUSA 1994 [1995?] ass. nov. (: 344) [IT, Sicilia]

Utricularietea intermedit-minoris

Helosciadio-Utricularietum exoletae GÉHU, KAABECHE et GHARZOULI 1994c ass. nov. (: 304) [DZ]

Wintero-Nothofagetea

O Racomitrio-Pilgerodendrienion PISANo 1994 suball. nov., nom. prov. (: 10) [art. 3b] [CL, Chile South]

O Hebetum ellipticae PISANO 1994 ass. nov., nom. prov. (: 9, 15) [art. 3b] [CL, Chile South]

O Hebetum salicifolii PISANO 1994 ass. nov., nom. prov. (: 9, 15) [art. 3b] [CL, Chile South]

O Racomitrio-Pilgerodendretum PISANO 1994 ass. nov., nom. prov. (: 10, 14) [art. 3b] [CL, Chile South] 
Incertae sedis

- Allenrolfeetum occidentalis Peinado, AlCaraz, Delgadillo, de la Cruz, Álvarez et AulRre 1994 ass. nov. (: 65) [MX, Mexico Northwest; US, Califomia]

O Alno glutinosae-Coryletum avellanae JULVE et GILLET 1994 ass. nov., nom. prov. (: 55) [art. 3b] [PL]

O Asplenio-Hedycaryetum baudouinii HoFF 1994 ass, nov., nom. prov. (: 684) [art. 3b] [NC]

O Athyrio filicis-feminae-Hyacinthoidetum non-scriptae DE FOUCAULT 1994a ass. nov., nom. inval. (: 7) [art. 5] [FR]

- Atriplici julaceae-Frankenietum palmeri Peinado, AlCARAZ, Delgadillo, DE la CRUZ, Álvarez et AuIRRE 1994 ass. nov. (: 65) [MX, Mexico Northwest; US, California]

- Axonopo schultesii-Schoenocephalietum martiani DUIVENvoORDEN et CLEEF 1994 ass. nov. (: 219) [CO]

- Bartramio potosicae-Bryoerythrophylletum jamesonii ALMEIDA, CLEEF, HERRERA, VELAZQUEZ et LUNA 1994 ass. nov. (: 401) [MX, Mexico Central]

O Basselenio-Araucarietum humboldtensis HoFf 1994 ass. nov., nom. prov. (: 685) [art. 3b] [NC]

O Beccariello-Rapaneetum lanceolatae HoF 1994 ass. nov., nom. prov. (: 685) [art. 3b] [NC]

O Betulo carpaticae-Pinetum sylvestris JULVE et GLLET 1994 ass. nov., nom. prov. (: 55) [art. 3b] [PL]

O Betulo humilis-Betulocoenetum pubescentis JULVE et GILLET 1994 coenass. nov., nom. prov. (: 57) [art. 3b, 3d] [PL]

O Betulo humilis-Salicetum rosmarinifoliae JULVE et GILLET 1994 ass. nov., nom. prov. (: 58) [art. 3b] [PL]

O Betulo pubescentis-Alnetum glutinosae JULVE et GILLET 1994 ass. nov., nom. prov. (: 57) [art. 3b] [PL]

O Betulo pubescentis-Piceetum abietis JULVE et GILLET 1994 ass. nov., nom. prov. (: 54) [art. 3b] [PL]

O Boehmerietum virgatae OHBA 1994 ass. nov., nom. inval. (: 31) [art. 5] [MP]

O Cardamino flexuosae-Rumicetum sanguinei JULVE et GILLET 1994 ass. nov., nom. prov. (: 48) [art. 3b] [PL]

- Carici bessarabicae-Festucetum orientalis GÉHU, ROMAN et BOULLET 1994 ass. nov. (: 80) [RO]

O Carici curtae-Quercocoenetum roboris JULVE et GLLLET 1994 coenass. nov., nom. inval. (: 52) [art. 3d] [PL]

O Carici elongatae-Alnocoenetum glutinosae JULve et GILLET 1994 coenass. nov., nom. inval. (: 55) [art. 3d] [PL]

O Carici lungentis-Salicetum lanatae SEKRETAREVA 1994 ass. nov., nom. inval. (: 62, 63) [art. 5] [RU, ]

O Carici remotae-Piceocoenetum abietis JULVE et GILLET 1994 coenass. nov., nom. inval, (: 51) [art. 3d] [PL]

O Carici sylvaticae-Orchidetum purpureae DE FOUCAULT 1994d ass. nov., nom. inval. (: 30, 32) [art. 5] [FR]

O Carpino betuli-Tilietum cordatae JuLve et GILLET 1994 ass. nov., nom. prov. (: 50) [art. 3b] [PL]

O Circaeo alpinae-Oxalidetum acetosellae JULVE et GILLET 1994 ass. nov., nom. prov. (: 56) [art. 3b] [PL]

O Cirsio oleracei-Fraxinocoenetum excelsioris JULVE et GILLET 1994 coenass. nov., nom. inval. (: 46) [art. 3d] [PL]

O Convolvulo arvensis-Imperatetum cylindrici ABD EL-GHANI 1994 ass. nov., nom. inval. (: 387, 390) [art. 5] [EG]

- Cymbopogono plurinodis-Eragrostietum gummifluae BEZUIDENHOUT, BREDENKAMP et THERON 1994b ass. nov. (: 159) [ZA, Transvaal]

- Cypero pannonici-Eleocharitetum quinqueflorae GÉHU, RoMAN et BOULLET 1994 ass. nov. (: 84) [RO]

O Dacridio-Araucarietum montanae HoFf 1994 ass. nov., nom. prov. (: 685) [art. 3b] [NC]

O Digitarietum gaudichaudii OHBA 1994 ass. nov., nom. inval. (: 36) [art. 5] [MP]

O Dryado integrifoliae-Salicetum lanatae SEKRETAREVA 1994 ass. nov., nom. inval. $(: 62,63)$ [art. 5] [RU, Magadan]

O Echinochloo-Melaleucetum quinquenerviae HOFF 1994 ass. nov., nom. prov. (: 685) [art. 3b] [NC]

- Elionuro mutici-Cymbopogonetum plurinodis BEZUIDENHOUT, BREDENKAMP et THERON 1994c ass. nov. (: 222) [ZA, Transvaal]

O Euphorbio-Stellarietum mediae ABD EL-GHANI 1994 ass. nov., nom. inval. (: 387, 396) [art. 5] [EG]

O Evonymo verrucosae-Daphnetum mezerei JULvE et GLLLET 1994 ass. nov., nom, prov. (: 51) [art. 3b] [PL]

O Excoecario-Sporoboletum virginici HoFf 1994 ass. nov., nom. prov. (: 685) [art. 3b] [NC]

- Falkio oblongae-Eragrostietum planae BEZUIDENHOUT, BREDENKAMP et THERON 1994c ass. nov. (: 223) [ZA, Transvaal]

- Festuco lividae-Arenarietum bryoidis AlMEIDA, CleEF, HerRERA, VelazQuEZ et LUNA 1994 ass. nov. (: 402) [MX, Mexico Central]

O Festuco sylvaticae-Tiliocoenetum cordatae JULVE et GILLET 1994 coenass. nov., nom. inval. (: 50) [art. 3d] [PL]

O Filipendulo denudatae-Alnocoenetum glutinosae JULVF et GILLET 1994 coenass. nov., nom. inval. (: 57) [art. 3d] [PL]

O Frangulo-Sorbetum aucupariae JULVE et GILLET 1994 ass. nov., nom. prov. (: 53) [art. 3b] [PL]

- Frankenio salinae-Juncetum acuti PEINADO, AlCARAZ, DElgadillo, DE LA CRUZ, ÁlVAREZ et AUIRRE 1994 ass. nov. (: 65) [MX, Mexico Northwest; US, California]

- Frankenio salinae-Sarcocornietum pacificae PEINADO, AlCARAZ, Delgadillo, de la Cruz, ÁlvarEZ et AUIRRE 1994 ass. nov. (: 64) [MX, Mexico Northwest; US, California]

O Fraxino excelsioris-Alnetum glutinosae JULVE et GILLET 1994 ass. nov., nom. prov. (: 47) [art. 3b] [PL]

O Glechomo hirsutae-Tiliocoenetum cordatae JULVE et GILLET 1994 coenass. nov., nom. inval. (: 50) [art. 3d] [PL]

O Hoyo-Ficetum prolixae HoFf 1994 ass. nov., nom. prov. (: 685) [art. 3b] [NC]

O Hoyo-Peperomietum HoFF 1994 ass. nov., nom. prov. (: 685) [art. 3b] [NC]

* Ilici aquifolii-Coryletum avellanae DE FoUCAULT 1994a ass. nov., nom. superfl. (: 7) [art. 29] [FR] 
- Jaumeo camosae-Distichlidetum spicatae PEINAdo, AlCARAZ, Delgadillo, DE LA CRUZ, Álvarez et AUIRre 1994 ass. nov. (: 65) [MX, Mexico Northwest; US, California]

- Lagunculario racemosae-Avicennietum germinantis PEINADO, AlCARAZ, Delgadillo, dE la CruZ, Álvarez et AUIRRE 1994 ass. nov. (: 64) [MX, Mexico Northwest]

- Lagunculario racemosae-Rhizophoretum mangle PEINADO, AlCARAZ, DElgadillo, DE LA CRUZ, ÁlVAREZ et AUIRRE 1994 ass. nov. (: 64) [MX, Mexico Northwest]

O Lamio maculati-Impatientetum noli-tangere JULVE et GILLET 1994 ass. nov., nom. prov. (: 47) [art. 3b] [PL]

O Ledo palustris-Pinocoenetum sylvestris JULVE et GILLET 1994 coenass. nov., nom. inval. (: 55) [art. 3d] [PL]

O Luzulo pilosae-Gymnocarpietum dryopteridis JULVE et GILLET 1994 ass. nov., nom. prov. (: 52) [art. 3b] [PL]

O Lycopodio annotini-Vaccinietum vitis-idaeae JULVE et GLLET 1994 ass. nov., nom. prov. (: 54) [art. 3b] [PL]

- Macaireo rufescentis-Bonnetietum martianae DuIvenvoORdEN et CLEEF 1994 ass. nov. (: 207) [CO]

- Maianthemo bifolii-Convallarietum majalis DE FouCAULT 1994a ass. nov. (: 12, 13) [FR]

O Metrosidero-Trichomanetum pallidi HoFf 1994 ass. nov., nom. prov. (: 685) [art. 3b] [NC]

- Monanthochloo littoralis-Arthrocnemetum subterminalis PEINADO, AlCARAZ, Delgadillo, DE LA CRUZ, ÁlvaREZ et AUIRRE 1994 ass. nov. (: 65) [MX, Mexico Northwest; US, California]

- Navio garcia-barrigae-Lagenocarpetum DUIVENVOORDEN et CLEEF 1994 ass. nov. (: 224) [CO]

O Parryo nudicaulis-Salicetum lanatae SEKRETAREVA 1994 ass. nov., nom. inval. (: 62, 63) [art. 5] [RU, Magadan]

- Paspalo dilatati-Hyparrhenietum hirtae BEZUIDENhOUT, BREDENKAMP et THERON 1994b ass. nov. (: 160) [ZA, Transvaal]

O Pemphiso-Kochietum hirsutae Hoff 1994 ass. nov., nom. prov. (: 685) [art. 3b] [NC]

$\Delta$ Phragmito-Thyphetum laxmanii (NEdELCU 1968) GÉHU, ROMAN et Boullet 1994 nom. nov. $(: 84,85)[$ RO]

O Phyllantho-Hedyotetum foetidae OHBA 1994 ass. nov., nom. inval. (: 31) [art. 5] [MP]

O Phyllanto-Homalietum deplanchei HoFf 1994 ass. nov., nom. prov. (: 685) [art. 36] [NC]

O Piceo abietis-Quercetum roboris JULVE et GILLET 1994 ass. nov., nom. prov. (: 51) [art. 3b] [PL]

- Polysticho setiferi-Asplenietum scolopendrii JULVE ex DE FoUCAULT 1994d ass. nov. (: 37, 38) [FR]

O Populo tremulae-Pinetum sylvestris JULVE et GILLET 1994 ass. nov., nom. prov. (: 53) [art. 3b] [PL]

O Portulacetum australis OHBA 1994 ass. nov., nom. inval. (: 44) [art. 5] [MP]

O Potentillo palustris-Betulocoenetum pubescentis JULVE et GILLET 1994 coenass. nov., nom. inval. (: 56) [art. 3d] [PL]

O Potentillo palustris-Scirpetum sylvatici JULve et GlLLET 1994 ass. nov., nom. prov. (: 57) [art. 3b] [PL]

- Pruno avii-Fraxinetum excelsioris DE FouCAuLT 1994d ass. nov. (: 29, 30) [FR]

- Querco petraeae-Betuletum albae DE FouCAULT 1994a ass. nov. (: 11) [FR]

O Ranunculo cassubici-Fraxinocoenetum excelsioris JULVE et GILLET 1994 coenass. nov., nom. inval. (: 47) [art. 3d] [PL]

- Roso arvensis-Viburnetum lantanae DE FouCAULT 1994d ass. nov. (: 30, 31) [FR]

- Salicomietum bigelovii PeInAdo, AlCaraz, Delgadillo, DE la CruZ, Álvarez et AUIRre 1994 ass. nov. (: 64) [MX, Mexico Northwest; US, California]

- Sarcocomietum pacificae Peinado, AlCARAZ, Delgadillo, DE la CruZ, Álvarez et Auirre 1994 ass. nov. (: 64 ) [MX, Mexico Northwest; US, California]

O Schino-Fimbristylidetum ferrugineae HoFF 1994 ass. nov., nom. prov. (: 685) [art. 3b] [NC]

O Semecarpo-Cordietum dichotomae HoFF 1994 ass. nov., nom. prov. (: 685) [art. 3b] [NC]

O Senecioni ovati-Myosotidetum sylvaticae DE FouCAuLT 1994a ass. nov., nom. prov. (: 11) [art. 3b] [FR]

O Sesuvietum portulacoidis OHBA 1994 ass. nov., nom. inval. (: 36, 37) [art. 5] [MP]

- Siphanthero hostmannii-Xyridetum paraensis DUIVENVOORDEN, CLEEF, MURILlO et ARBELAEZ in DUIVENVOORDEN et ClEEF 1994 ass. nov. (: 222) [CO]

- Spartinetum foliosae Peinado, AlCaraz, Delgadillo, DE la CruZ, Álvarez et Auirre 1994 ass. nov. (: 64) [MX, Mexico Northwest; US, California]

O Sphagno girgensohnii-Piceocoenetum abietis JULVE et GILLET 1994 coenass. nov., nom. inval. (: 54) [art. 3d] [PL]

O Spinifici littorei-Viticetum ovatae DE FOUCAULT 1994c ass. nov., nom. prov. (: 29) [art. 3b] [ID, ]

- Suaedetum moquinii Peinado, AlCaraz, Delgadillo, de la Cruz, Álvarez et Auirre 1994 ass. nov. (: 65) [MX, Mexico Northwest; US, California]

O Ribetum rubro-nigri JULVE et GILLET 1994 ass. nov., nom. prov. (: 47) [art. 3b] [PL]

O Trientali europaei-Pinocoenetum sylvestris JULVE et GILLET 1994 coenass. nov., nom. inval. (: 53) [art. 3d] [PL]

O Ulmo glabrae-Coryletum avellanae JULVE et GILLET 1994 ass. nov., nom. prov. (: 50) [art. 3b] [PL]

- Utricularietum neottioidis Duivenvoorden, CleEF, MURILlo et ARbElaEz in Duivenvoorden et CleEF 1994 ass. nov. (: 225) [CO]

- Xyrido wurdackii-Paspaletum tillettii DUIVENvOORDEN et CleEF 1994 ass. nov. (: 222) [CO] 


\section{REFERENCES}

Abbas H. \& GéHu J.-M. (1994): Colloq. Phytosoc. 22: 111-123.

ABD EL-GHANI M.M. (1994): Feddes Repert. 105: 387-398.

almeida L., Cleef A. M., Herrera A., Velazquez A. \& Luna I. (1994): Phytocoenologia 22: 391-436.

ASENSi A., Díez-Garretas B. \& MARTíN OSORIO V.E. (1994): Ecol, Medit. 19: 1-7.

BARBERO M. \& QUÉZEL P. (1994): Ann. Bot. (Rome) 52: 81-133.

BaRKMAN J.J., MORAVEC J. \& RAUSCHERT S. (1986): Code of phytosociological nomenclature. Vegetatio 67: 145-195.

Bartolo G., Brullo S. \& Pulvirenti S. (1994a) [1995?]): Boll. Acad. Gioenia Sci. Nat. Catania 27: 399-407.

Bartolo G., Brullo S. \& Pulvirenti S. (1994b) [1995?]): Boll. Acad. Gioenia Sci. Nat. Catania 27: 409-414.

BEHÇET L. (1994a): Turkish J. Bot. 18: 229-243.

BEHÇET L. (1994b): Turkish J. Bot. 18: 289-303.

BEHÇET L. (1994c): Turkish J. Bot. 18: 305-312.

BENABID A. \& FENNANE M. (1994): Lazaroa 14: 21-97.

BERNÁTOVÁ D. \& Uhlírová J. (1994): Biologia (Bratislava) 49: 1-11.

BEZUIDENHOUT H., BREDENKAMP G.J. \& THERON G.K. (1994a): S. African J. Bot. 60: 72-81.

BEZUiDENHOUT H., BREDENKAMP G.J. \& THERON G.K. (1994b): S. African J. Bot. 60: 152-161.

BEZUIDENHOUT H., BREDENKAMP G.J. \& THERON G.K. (1994c): S. African J. Bot. 60: 214-224.

BeZuidenhout H., BredenKamp G.J. \& Theron G.K. (1994d): Koedoe (Pretoria) 37: 1-18.

BIONDI E., AllegGrEZZA M., TAFFETANI F. \& WILDPRET DE LA TORRE W. (1994): Fitosociologia 27: 107-121.

Biondi E., Ballelli S., Allegrezza M., Taffetani F. \& Francalancia C. (1994): Fitosociologia 27: 51-66.

Biondi E., Blasi C., Brugiapaglia E., Fogu M.C. \& Mossa L. (1994): Allionia 32: 303-323.

BIORET F. (1994): Colloq. Phytosoc. 22: 126-144.

BiURRUn I., Garcia-Mijangos I. \& LoIDI J. (1994): Bot. Helv. 104: 31-54.

Botineau M. \& GHesteM A. (1994): Colloq. Phytosoc. 22: 333-346.

Braque R. \& Loiseau J.-E. (1994): Bull. Soc. Bot. Centre-Ouest, N.S., Numéro Spécial 12: 1-193.

BRICAUD O. \& ROUX C. (1994): Lichenologist 26: 113-134.

BRULLO S., Minissale P. \& SPAMPINATO G. (1994): Fitosociologia 27: 5-50.

Brullo S., Scelsi F. \& Siracusa G. (1994) [1995?]. Boll. Acad. Gioenia Sci. Nat. Catania 27: 341-365.

Cano Carmona E. \& García Fuentes A. (1994): Monogr. Fl. Veg. Bética 7-8: 35-76.

CAPELo J.H., COSTA J.C. \& Lousã M.F. (1994): Anais Inst. Super. Agron. 44: 285-301.

Čarni A. (1994): Colloq. Phytosoc. 22: 467-497.

ChIFU T. \& ŞזEFAn N. (1994): Analele Sti.. Univ. Al. I. Cuza laşi, Sect. 2. 40: 71-80.

CHYTRÝ M. (1994): Scripta Fac. Sci. Nat. Univ. Masaryk. Brun. Biol. 22-23: 121-134.

Copull lard P., Gueugnot J., Julve P., Michalet R. \& Michelin Y. (1994): Ecol. Medit. 20: 9-57.

Costa J.C., Capelo J.H., Lousã M.F. \& AGUiAR C. (1994): Colloq. Phytosoc. 22: 499-526.

DE LA TORRE A. \& ALCARAZ F. (1994): Lazaroa 14: 125-138.

Delelis-Dusollier A., Botineau M., WatTEZ-Franger A. \& GHestem A. (1994): Acta Bot. Gallica 140: 507-517.

DelpeCH R. (1994): Colloq. Phytosoc. 22; 347-395.

DENGLER J. (1994): Gleditschia 22: 179-321.

Díaz González T.E. \& FeRnández PRIETo J.A. (1994): Itinera Geoboi. 8: 243-528.

Déz Garretas B. \& Asensi MARFIL A. (1994): Colloq. Phytosoc. 22: 539-553.

DUIVENVOORDEN J.F. \& CLEEF A.M. (1994): Phytocoenologia 24: 197-232.

EgEa J.M. \& Llimona X. (1994): Bull. Soc. Linn. Provence 45: 263-281.

EGGENSBERger P. (1994): Ber. Bayer. Bot. Ges., Beih. 8: 1-239.

FEoli Chiapella L. \& Poldini L. (1994): Stud. Geobot. 13: 3-140.

Foucault B. DE (1994a): Bull. Soc. Bot. N. France 47 (2): 5-20.

Foucault B. DE (1994b): Bull. Soc. Bot. N. France 47 (2): 21-24.

Foucault B. DE (1994c): Bull. Soc. Bot. N. France 47 (2): 29-39.

FouCAuLt B. DE (1994d): Bull. Soc. Bot. N. France 47 (3): 29-39.

Foucault B. DE (1994e): Colloq. Phytosoc. 22: 431-455.

FREY W. \& KÜRSCHNER H. (1994): Nova Hedwigia 59: 365-378.

GAFTA D. (1994): Braun-Blanquetia 12.

GALÁN DE MERA A. Naturalia Baetica 6: 113-123.

García Mijangos I., Loidi J. \& Herrera Gallastegui M. (1994): Lazaroa 14: 99-110.

García Río R. \& NaVARRo ANDRÉs F. (1994): Stud. Bot. 12: 23-202.

GÉHU J.-M. (1994): Colloq. Phytosoc. 22: 183-212.

GéHU J.-M. \& BIONDI E. (1994): Braun-Blanquetia 13: 1-149.

GÉHu J.-M. \& BOURNiQue C. (1994): Bull. Soc. Bot. Centre-Ouest, N.S. 25: 99-102.

Géhu J.-M., KAABeche M. \& Gharzouli R. (1994a): Phytocoenologia 24: 369-382.

GÉHU J.-M., KaAbeche M. \& Gharzouli R. (1994b): Fitosociologia 27: 67-71. 
Géhu J.-M., KaAbeche M. \& Gharzouli R. (1994c): Colloq. Phytosoc. 22: 297-329.

GÉHU J.-M., ROMAN N., BOULleT V. (1994): Fitosociologia 27: 73-89.

Giaccone, G., Alongi G., Pizzuto F. \& Cossu A. (1994) [1995?]. Boll. Accad. Gioenia Sci. Nat. Catania 27: $111-157$.

GŁAZzK T. \& ŁuSzCZynSKA B. (1994): Fragm. Florist. Geobot. 39: 277-290.

GoluB V.B. (1994a): Folia Geobot. Phytotax. 29: 15-54.

GoluB V.B. (1994b): Feddes Repert. 105: 499-515.

Hoff M. (1994): Colloq. Phytosoc. 22: 663-688.

Hollis S. \& BRUMMITT R.K. (1992): World geographical scheme for recording plant distributions. Hunt Institute for Botanical Documentation, Carnegie Mellon Univ., Pittsburg.

JEHLÍK V. (1994): Ber. Reinhold-Tüxen-Ges. 6: 235-278.

Julve P. \& Gillet F. (1994): Phytocoenosis N.S. 6, Suppl. Cartogr. Geobot. 4: 45-66.

Kaabeche M. \& Gharzouli R. (1994): Colloq. Phytosoc. 22: 596-609.

KIELLAND-LUND J. (1994): Phytocoenologia 24: 299-310.

Kim M.-H., Hukusima T. \& Hoshino Y. (1994): J. Phytogeogr. Taxon. 42; 61-73.

KLEIN J.-C. \& LACOSTE A. (1994): Phytocoenologia 24: 401-421.

KolbeK J. \& SÁdlo J. (1994): Preslia 66: 115-131.

KorolyUK A.J. \& NAMZALOV B.B. (1994): Siberian J. Ecol. 1: 467-473.

KOWARIK I. \& LANGER A. (1994): Verh. Bot. Vereins Berlin Brandenburg 127: 5-43.

Kukovitsa G.S., Movchan Y.I., SOloMakha V.A. \& SHElYag-Sosonko Y.R. (1994): Ukrayins'k. Bot. Zhum. 51(2-3): 35-48.

Ladero Álvarez M., Valle Gutiérez C.J. \& Gutiérez Balbas A. (1994): Candollea 49: 499-507.

Loidi J., Herrera M., Olano J.M. \& Silvan F. (1994): J. Veg. Sci. 5: 533-540.

Lous̃̃ M., Esprito Santo M.D. \& CosTA J.C. (1994): Anais Inst. Super. Agron. 44: 215-233.

MEDDOUR R. (1994): Ecol. Medit. 19: 43-51.

Michalet R. \& Philippe T. (1994): Colloq. Phytosoc. 22: 397-430.

Mota J.F., Cabello J., Gómez F. \& Peñas J. (1994): Ecol. Medit. 19: 53-60.

MoYa J.J., Ríos R.M. \& GuerRa J. (1994): Cryptog. Bryol. Lichénol. 6: 43-65.

OCAKVERDI H. (1994): Turkish J. Bot. 18: 245-265.

OHBA T. (1994): Nat. Hist. Res., Special Issue 1: 13-69.

PARADIS G. \& LORENZONI C. (1994): Monde Pl. 449: 19-26.

PASSARGE H. (1994a): Phytocoenologia 24: 337-367.

PASSARGE H. (1994b): Ber. Bayer. Bot. Ges. 64: 165-188.

PASSARGE H. (1994c): Tuexenia 14: 83-111.

PEDROTTI F. (ed.) (1994): Guida all'escursione della Società Italiana di Fitosociologia in Trentino (1-5 luglio 1994). Camerino.

Peinado M., Alcaraz F., Delgadilo J., de la Cruz M., Álvarez J. \& Auirre J.L. (1994): Vegetatio $110: 55-66$. PETRICCIONE B. (1994): Fitosociologia 26: 189-199.

Piazza C. \& Paradis G. (1994): Bull. Soc. Bot. Centre-Ouest, N.S. 25: 59-98.

Pignatti E. \& Pignatti S. (1994): J. Veg. Sci. 5: 55-62.

PISANo E. (1994): Anales Inst. Patagonia, Ci. Nat. 21: 5-33.

Poldini L. \& MARTINI F. (1994): Stud. Geobot. 13: 141-214.

POLDINI L. \& NARDINI S. (1994): Stud. Geobot. 13: 215-298.

Preising E., Vahle H.-C., BRAndes D., Hofmeister H., TUXen J. \& Weber H.-E. (1994): Naturschutz Landschaftspflege Niedersachsen 20 (7-8): 1-163.

PUGLISI M. (1994) [1995?]: Boll. Soc. Acad. Gionea Sci. Nat. Catania 27: 79-91.

Quézel P., Barbero M., Benabid A. \& Rivas-MarTínez S. (1994): Phytocoenologia 22: 537-582.

RINGER C. (1994): Drosera '94: 125-137.

Roselló Gimeno R. (1994): Catálogo florístico y vegetación de la comarca natural del Alto Mijares (Castellon). Diputació de Castelló, Castelló.

Sanda V., Popescu A. \& Serbanescu G. (1994): Rev. Roumaine Biol., Sér. Biol. Vég. 39: 11-19.

SCHÖNFELDER P. \& VOGGENREITER V. (1994): Phytocoenologia 24: 461-493.

SEKretareva N.A. (1994): Bot. Zhurn. (Moscow \& Leningrad) 79 (12): $58-64$.

SERIN M. \& EYCE B. (1994): Turkish J. Bot. 18: 201-227.

SINSIN B. (1994): Belg. J. Bot. 127: 87-103.

ŞTEFAn N. (1994): Anale Ş̧ıi., Univ. Al. I. Cuza Iaşi, Ser. Nouă, 2.a. 39: 77-80.

STEIGER P. (1994): Wälder der Schweiz. Ott, Thun.

TATli Â., EYCE B. \& SERIN M. (1994): Turkish J. Bot. 18: 267-288.

THEURILLAT J.-P. (1997): Index of proposals to correct, to inverse, to reject, and to conserve names of syntaxa in 1993. Bot. Helv. 107: 263-269. 
Theurillat J.-P. \& MoraveC J. (1990): Index of new names of syntaxa published in 1987. Folia Geobot. Phytotax. 26: 79-99.

Theurillat J.-P. \& Moravec J. (1992): Index of new names of syntaxa published in 1989. Folia Geobot. Phytotax. 27: 69-101.

Theurillat J.-P. \& Moravec J. (1994): Index of new names of syntaxa published in 1991. Folia Geobot. Phytotax. 29: $385-412$.

TheuRILlat J.-P. \& MORAVEC J. (1995): Index of new names of syntaxa published in 1992. Folia Geobot. Phytotax. 30: $331-362$.

Theurillat J.-P. \& Moravec J. (1996): Index of new names of syntaxa published in 1993. Folia Geobot. Phytotax. 31: 473-516.

TOMASELli M. (1994): Fitosociologia 26: 35-50.

TOMASELLI M. \& ROSSI G. (1994): Fitosociologia 26: 51-62.

VADAM J.-C. (1994): Cryptog., Bryol. Lichénol. 15: 153-159.

VALSECCHI F. (1994): Fitosociologia 27: 127-138.

VARESe P. (1994): Colloq. Phytosoc. 22: 565-593.

VANDEN BERGHEN C. (1994): Lejeunia N.S. 144: 1-26.

WIBKIRCHEN R. \& KRAUSE S. (1994): Tuexenia 14: 425-444.

WOLFF P., DIEKIOBST H. \& SCHWARZER A. (1994): Tuexenia 14: 343-380.

ZECHMEISTER H. \& MUCINA L. (1994): J. Veg. Sci. 5: 384-402.

\section{ADDITION TO INDEX 1991}

Asplenietea trichomanis

O Potentillo doerflerii-Juncetum irifidi STEVANOvić, JovaNOvić et LAKUSić 1991 ass, nov., nom. inval. (: 80) [art. 5] [YU]

Brachypodio pinnati-Betuletea

- Brachypodio pinnati-Betuletea ERMAKOV, KOROLYUK et LATCHINSKY 1991 cl. nov. (: 8) [RU, Southern Siberia]

- Calamagrostio epigeï-Betuletalia pendulae KOROLYUK in ERMAKOV, KOROLYUK et LATCHINSKY 1991 ord. nov. (: 28) [RU, Southem Siberia]

- Carici macrourae-Pinetalia sylvestris ERMAKov, KoROLYUK et LATCHINSKY 199] ord. nov. (: 13) [RU, Southern Siberia]

- Carici pediformis-Laricetalia sibiricae ERMAKOV in ERMAKOV, KOROLYUK et LATCHINSKY 1991 ord. nov. (31) [RU, Southem Siberia]

- Calamagrostio epigeii-Betulion pendulae KOROLYUK in ERMAKOV, KOROYUK et LATCHINSKY 1991 all. nov. (: 28) [RU, Southem Siberia]

- Carici pediformis-Laricion sibiricae ERMAKOv in ERMAKOV, KOROLYUK et LATCHINSKY 1991 all. nov. (: 31) [RU, Southern Siberia]

- Lathyro gmelinii-Pinion sylvestris ERMAKOV in ERMAKOV, KOROLYUK et LATCHINSKY 1991 all. nov. (: 23) [RU, Southem Siberia]

- Vicio unijugae-Pinion sylvestris ERMAKov, KOROLYUK et LATCHINSKY 1991 all. nov. (: 14) [RU, Southem Siberia]

- Achyrophoro-Pinenion ERMAKOv et LATCHINSKY in ERMAKOV, KOROLYUK et LATCHINSKY 1991 suball. nov. (: 15) [RU, Southern Siberia]

- Carici macrourae-Pinenion sylvestris ERMAKOV in ERMAKOV, KOROLYUK et LATCHINSKY 1991 suball. nov. (: 26) [RU, Southern Siberia]

- Cruciato krylovii-Pinenion sylvestris ERMAKov in ÉRMAKov, KOROLYUK et LATCHINSKY 1991 suball. nov. (: 19) [RU, Southern Siberia]

- Geranio albiflori-Pinenion sylvestris ERMAKOV in ERMAKov, KOROLYUK et LATCHINSKY 1991 suball. nov. (: 24) [RU, Southern Siberia]

- Vicio unijugae-Pinenion sylvestris Korolyuk in ERMaKov, KOROLYUK et LaTCHINSKY 1991 suball. nov. (: 22) [RU, Southern Siberia]

- Anemonoido caeruleae-Pinetum sylvestris ERMAKov in ERMAKOv, KOROlYUK et LatCHINSKY 1991 ass. nov. (: 20 , 47) [RU, Southern Siberia]

- Artemisio macranthae-Pinetum sylvestris LATCHINSKY in ERMAKOV, KOROLYUK et LATCHINSKY 1991 ass. nov. (: 18, 41) [RU, Southern Siberia]

- Astragalo glycyphylli-Pinetum sylvestris KOROLYUK in ERMAKOV, KOROLYUK et LATCHINSKY 1991 ass. nov. (: 21 , 53) [RU, Southern Siberia] 
- Caragano fruticis-Pinetum sylvestris ERMAKOV in ERMAKOV, KOROLYUK et LATCHINSKY 1991 ass. nov. (: 26, 61) [RU, Southern Siberia]

- Carici pediformis-Laricetum sibiricae ERMAKov in ERMAKOv, KOROLYUK et LATCHINSKY 1991 ass. nov. (: 32, 79) [RU, Southern Siberia]

- Cirsio heterophylli-Betuletum pendulae KOROLYUK in ERMAKOV, KOROLYUK et LATCHINSKY 1991 ass. nov. (: 31 , 75) [RU, Southern Siberia]

- Cnidio dubii-Pinetum sylvestris LATCHINSKY in ERMAKOv, KOROLYUK et LATCHINSKY 1991 ass. nov. (: 19, 44) [RU, Southern Siberia]

- Crepido praemorsae-Pinetum sylvestris ERMAKOv in ERMAKOV, KOROLYUK et LATCHINSKY 1991 ass. nov. (: 16, 36) [RU, Southern Siberia]

- Dentario sibiricae-Pinetum sylvestris ERMAKOV in ERMAKOV, KOROLYUK et LATCHINSKY 1991 ass. nov. (: 25, 58) [RU, Southern Siberia]

- Festuco ovinae-Pinetum sylvestris ERMAKov in ERMAKOv, KOROLYUK et LATCHINSKY 1991 ass. nov. (: 17, 39) [RU, Southern Siberia]

- Filipendulo vulgaris-Pinetum sylvestris ERMaKov in ERMaKov, KorolyuK et LATCHINSKY 1991 ass. nov. (: 21 , 50) [RU, Southern Siberia]

- Matteuccio-Pinetum sylvestris ERMAKOV in ERMAKOV, KOROLYUK et LATCHINSKY 1991 ass. nov. (: 28, 66) [RU, Southem Siberia]

- Peucedano morisonii-Betuletum pendulae KOROLyUK in ERMAKov, KOROLYUK et LATCHINSKY 1991 ass. nov. (: 29 , 69) [RU, Southern Siberia]

- Phalarido-Betuletum pendulae KOROLYUK in ERMAKOV, KOROLYUK et LATCHINSKY 1991 ass. nov. (: 30, 73) [RU, Southern Siberia]

- Poo urssulensis-Betuletum pendulae KOROLYUK in ERMAKOV, KOROLYUK et LATCHINSKY 1991 ass. nov. (: 29, 71$)$ [RU, Southern Siberia]

- Spiraeo salicifoliae-Pinetum sylvestris ERMAKov in ERMAKOV, KoROLYUK et LATCHINSKY 1991 ass. nov. (: 27, 63) [RU, Southern Siberia]

- Thesio repentis-Pinetum sylvestris ERMAKov in ERMAKOV, KOROLYUK et LATCHINSKY 1991 ass. nov. $(: 15,33)$ [RU, Southern Siberia]

Festucetea vaginatae

O Festuco duriusculae-Euphorbietum glabrifoliae JOVANOVIĆ-DUNJIĆ et JOVANOVIĆ 1991 ass. nov., nom. prov. (: 72) [art. 3b] [YU]

Incertae sedis

O Polygono punctati-Rumicetum paraguyensis EsCHKUCHE et FonTANA in FONTANA 1991 ass. nov., nom. prov. (: 11) [art. 3b] [AR, Argentina Northeast]

\section{REFERENCES}

ERMAKOV N.B., KoROLYUK A.YU. \& LATCHINSKY N.N. (jun.) (1991): Floristicheskaya klassifikatsiya mezofilnykh travyanykh lesov Yuzhnoi Sibiri. Novosibirsk.

FonTANA J.L. (1991): Folia Bot. Geobot. Corrent. 6: 1-17.

JovanoviĆ-DunjIĆ R. \& Jovanović S. (1991): Glasn. Bot. Zavoda Baste Univ. Beogradu 23: 69-75.

Stevanović V., Jovanović S. \& Lakusić D. (1991): Glasn. Bot. Zavoda Baste Univ. Beogradu 23: 77-84.

\section{ADDITION TO INDEX 1992}

Agropyretea repentis

- Calamagrostietum epigeios KostLlov in SOLOMAKHA, KostILOv et SHELYAG-SOSONKo 1992 ass. nov. (: 125) [UA]

- Elytrigio repentis-Lycietum barbati Kostllov in SOlOMAKHA, Kostllov et SHElyag-Sosonko 1992 ass. nov. (: 127) [UA]

Artemisietea vulgaris

O Ambrosio artemisifoliae-Xanthietum strumariae KOSTLOV in SOLOMAKHA, KOSTILOV et SHELYAG-SOSONKO 1992 ass. nov. nom. inval. (: 162) [art. 5] [UA]

- Chaerophyllo hirsuti-Cirsietum oleracei Kostilov in SOLOMAKHA, Kostllov et Shelyag-Sosonko 1992 ass. nov. (: 158) [UA] 
- Urtico dioicae-Bryonietum albae Kostilov in SOLOMAKHA, Kostilov et SHELYAG-SOSONKo 1992 ass. nov. (: 152,155$)$ [UA]

- Urtico dioicae-Tanacetetum vulgaris KostlLov in SOLOMAKHA, KOSTILOV et SHELYAG-SOSONKO 1992 ass. nov (: 152) [UA]

Chenopodietea

- Arctio lappae-Chenopodietum albae Kostilov in SOlOMAKHA, Kostilov et Shelyag-Sosonko 1992 ass. nov. (: 175) [UA]

- Rumici conferti-Galeopsietum speciosae Kostilov in SOlOMAKHA, Kostllov et SHELYAG-Sosonko 1992 ass. nov. (: 177) [UA]

Meliloto-Artemisietea absinthii

- Achilleo millefolii-Grindelietum squarrosae Kostilov in SOLOMAKHA, KostILOv et SHELYAG-SOSONKO 1992 ass. nov. (: 197) [UA]

- Pastinaco sativae-Daucetum carotae Kostilov in SolomaKha, Kostilov et Shelyag-Sosonko 1992 ass. nov. (: 194) [UA]

Secalietea

- Hibisci trioni-Chenopodietum polyspermi SOlOMAKHA in SOLOMAKHA, KostILOV et SHELYAG-SOSONKO 1992 ass. nov. (: 59) [UA]

- Lamio purpurei-Cerastietum holosteoidis (GAMOR et al. 1985) SOlOMAKHA in SOLOMAKHA, KoSTLOV et SHELYAG-SOSONKo 1992 ass. nov. (: 60) [UA]

O Polygono scabri-Galinsogetum ciliatae SolomakHa in SolomakHa, Kostilov et Shelyag-Sosonko 1992 ass. nov., nom. inval. (: 61) [art. 3f] [UA]

Sisymbrio-Onopordetea

- Aegilopo biuncialis-Avenetum persicae Kostilov in SOLOMAKHA, KOStllov et SHELYAG-SOSONKo 1992 ass. nov. (: 225) [UA]

- Bromo squarrosi-Sonchetum oleracei KOStIlov in SOLOMAKHA, Kostilov el SHELYAG-SosonKo 1992 ass. nov. (: 225) [UA]

- Chamomillo recutitae-Malvetum mauritianae KosTILOV in SOLOMAKHA, KOSTILOV et SHELYAG-SOSONKO 1992 ass. nov. (: 224) [UA]

- Diplotaxio muralis-Malvetum erectae Kostilov in SOlomaKHA, Kostilov et ShelyaG-Sosonko 1992 ass. nov. (: 227) [UA]

- Hordeo murini-Peganetum harmalae Kostilov in SOlomakha, Kostllov et SHElyaG-Sosonko 1992 ass. nov. (: 226) [UA]

\section{REFERENCES}

SOlomakha V.A., Kostilov O.V. \& ShelyaG-SoSONKo Y.R. (1992): Synantropna roslinnist Ukrainy. Kyiv.

\section{ADDITION TO INDEX 1993}

Alnetea glutinosae

- Carici aquatilis-Salicetum lapponi TARAN 1993b ass. nov. (: 80) [RU, Western Siberia]

- Carici juncellae-Salicetum rosmarinifoliae KOROLYUK et TARAN in TARAN 1993b ass. nov. (: 80) [RU, Western Siberia]

- Sanguisorbo officinalis-Salicetum rosmarinifoliae TARAN 1993b ass. nov. (: 82) [RU, Western Siberia]

Asplenietea trichomanis

- Asplenietum marini Gamisans et PaRAdIs 1993 ass. nov. (: 36) [FR, Corse]

O Carici laevis-Leontopodietum alpini STANIĆ et LAKUSIĆ 1993 ass. nov., nom. inval. (: 23) [art. 5] [YU]

- Cymbalarietum aequitrilobae GAMISANS et PARADIS 1993 ass. nov. (: 11) [FR, Corse]

O Edraiantho jugoslavici-Hieracietum humilis STANIĆ et LAKUSIĆ 1993 ass. nov., nom. inval. (: 22) [art. 5] [YU]

- Micromerio filiformis-Allietum antoni-bolosii RITA et BIBILONI 1993 ass. nov. (: 227, 228) [ES, Baleares] 
Greenovio-A eonietea

- Aeonietum lindleyi Voggenreiter ex Rivas-Martínez, WildPret DE LA TORRE, DEl ARCo AgULLAR, Rodriguez, Pérez de Praz, García-Gallo, Acebes Ginovés, díaz González et Fernández-González 1993 ass. nov. (: 309) [ES, Canary Is]

Is oeto-Nanojuncetea

- Marisco hamulosi-Crypsietum schoenoidis TARAN 1993a ass. nov. (: 82) [RU, Western Siberia]

Kleinio-Euphorbietea canariensis

- Mayteno-Juniperion canariensis SANTOS et FERNÁNDEZ GALVÁN in SANTOS 1983 cort. RIVAS-MARTínEZ, Wildpret de la Torre, del arco aguilar, Rodríguez, Pérez de Praz, García-Gallo, acebes Ginovés, DÍAZ GONZÁLEZ et FERNÁNDEZ-GONZÁLEZ 1993 (: 198)

- Brachypodio arbusculae-Juniperetum canariensis FERNÁNDEZ GALVÁN 1983 corr. RIVAS-MARTÍNEZ, WILDPRET DE la Torre, del Arco Aguilar, Rodríguez, Pérez de Praz, García-Gallo, Acebes Ginovés, Díaz GONZÁlEZ et FERNÁNDEZ-GONZÁlEZ 1993 (: 199)

- Euphorbietum bertheloto-canariensis RIVAS-MARTínEZ, WILDPRET DE LA TORRE, DEL ARCo AGULLAR, RODRÍGUEZ, PÉrez de Praz, García-Gallo, Acebes Ginovés, díaz González et Fernández-González 1993 ass. nov. (: 191) [ES, Canary Is]

$\triangle$ Rubio fruticosae-Juniperetum canariensis SANTOS in RIVAS-MARTíNEZ, WILDPRET DE LA TORRE, DEL ARCO agullar, Rodríguez, Pérez de Praz, García-Gallo, acebes Ginovés, díaz González et FERNÁNDEZ-GONZÁlEZ 1993 ass. nov. (: 199) [ES, Canary Is]

Koelerio-Corynephoretea

- Sileno-Tortuletum DoING 1993 ass. nov. (: 43) [NL]

Pegano-Salsoletea

- Ephedro fragilis-Withanietum frutescentis RITA et BIBILONI 1993 ass. nov. (: 236, 237) [ES, Baleares]

Pruno-Lauretea azoricae

O Salicion canariensis Rivas-Martínez, WildPret de la TORRe, del ArCo Agullar, Rodríguez, PÉrez de PRAZ, García-Gallo, ACEBes Ginovés, Díaz Gonz.Ález et FernándeZ-GonzÁlez 1993 ass. nov., nom. prov. (: 225) [art. 3b] [ES, Canary Is]

Quercetea ilicis

- Ferulo communis-Juniperetum phoeniceae RITA et BIBILONI 1993 ass. nov. (: 219) [ES, Baleares]

Salicetea purpureae

- Anemonidio-Populetum nigrae TARAN 1993c ass. nov. (: 85 ) [RU, Western Siberia]

- Salicetum dasycladi TARAN 1993c ass. nov. (: 85) [[RU, Western Siberia]

- Spiraeo salicifoliae-Populetum tremulae TARAN 1993c ass. nov. (: 85) [[RU, Westem Siberia]

\section{REFERENCES}

DOING H. (1993): Stratiotes 6: 40-52.

Gamisans J. \& Paradis G. (1993): Trav. Sci. Parc Nat. Rég. Réserves Nat. Corse 37: 1-68.

Rita J. \& BibILONi G. (1993): La vegetació. In: Alcover J.A., Ballesteros E. \& Fornós J.J. (eds.), História natural de l'Arxipèlago de Cabirera, Moll, Palma de Mallorca, pp. 207-255.

Rivas-Martínez S., Wildpret de la Torre W., Arco agullar M. Del, Rodríguez O., Pérez de Praz P.L., García-Gallo A., ACEBes Ginovés J.R., Díaz GonzÁlez T.E. \& FERnÁndez-GonzÁlez F. (1993): Itinera Geobot. 7: $169-374$.

Stanić S. \& Lakusić D. (1993): Glasn. Bot. Zavoda Baste Univ. Beogradu 24-25: 21-31.

TARAN G.S. (1993a): Siberian J. Biol. 1993 (5): 79-84.

TARAN G.S. (1993b): Siberian J. Biol, 1993 (6): 79-84.

TARAN G.S. (1993c): Siberian J. Biol 1993 (6): 85-91. 